MATHEMATICS OF COMPUTATION

Volume 78, Number 268, October 2009, Pages 2019-2046

S 0025-5718(09) 02229-7

Article electronically published on February 11, 2009

\title{
FIRST DERIVATIVES ESTIMATES FOR FINITE-DIFFERENCE SCHEMES
}

\author{
ISTVÁN GYÖNGY AND NICOLAI KRYLOV
}

\begin{abstract}
We give sufficient conditions under which solutions of discretized in space second-order parabolic and elliptic equations, perhaps degenerate, admit estimates of the first derivatives in the space variables independent of the mesh size.
\end{abstract}

\section{INTRODUCTION}

This is the first article out of a series of three devoted to estimating space derivatives of solutions of discretized in space second-order parabolic and elliptic equations. We allow equations to degenerate and to become just first-order equations. In the present article we only deal with the first-order derivatives. In the second part of this project we will prove higher-order derivatives estimates and in the third part apply them to showing a method of accelerating finite-difference approximations to any given rate for equations in the whole space.

Numerical approximations for linear and quasilinear partial differential equations is a rather old and well-developed area. We refer the reader to [2] and the references therein, following which one can track down original papers by D. Aronson, L. Bers, R. Courant-K. Friedrichs-H. Lewy, J. Douglas, F. John, O. Ladyzhenskaya, P. Lax, H. Levy, L. Liusternik, I. Petrovskii, and many many others to which we only add [8] and two more papers [4] and [10] where discrete methods are applied to approximate stochastic partial differential equations.

A major difference of this article from all the above-mentioned ones is that we focus on investigating the smoothness of approximating solutions rather than on convergence only. For each point $x \in \mathbb{R}^{d}$ we move the original grid in such a way that $x$ becomes a grid point. This allows us to define the approximate solution in all of $\mathbb{R}^{d}$ rather than only on the grid, and we investigate how smooth the approximating solution is with respect to $x$. We estimate true derivatives rather than their difference approximations.

Estimating the sup norms of the first-order derivatives for solutions of finitedifference schemes for linear and fully nonlinear second-order degenerate equations plays a major role in estimating the rate of convergence of approximating solutions to the true solution in the sup norm. The most general results for fully nonlinear equations concerning the rates can be found in [3] and in the references therein. A

Received by the editor January 22, 2008 and, in revised form, September 26, 2008

2000 Mathematics Subject Classification. Primary 65M06, 39A70.

Key words and phrases. Cauchy problem, finite differences, first derivatives estimates.

The work of the second author was partially supported by NSF grant DMS-0653121.

(C)2009 American Mathematical Society Reverts to public domain 28 years from publication 
recent development in the issue of estimating the Lipschitz constant and secondorder differences for approximating solutions for fully nonlinear equations without applications to estimating the rate of convergence is presented in [6]. Previously, the Lipschitz constants and higher-order derivatives estimates were obtained in 2 for time-space discretization of linear degenerate parabolic equations and in [1] for fully nonlinear equations. They are also applied to estimating the rate of convergence. In a sense the present article is close to 2 . However, here we only deal with the first-order derivatives estimates and for equations discretized only in the space variable. We introduce a new type of sufficient conditions for obtaining the estimates (see Assumption 2.3 and the discussion in Section 5). These conditions are much weaker and more detailed than the corresponding ones in [2]. Our method is also somewhat different. Instead of considering just the sum of squares of the difference increments along the mesh, we add to it the square of the full gradient with a small constant factor. This allows us to estimate the gradient.

In this connection it is worth noting that such an estimate for finite-difference approximations of the first-order directional derivatives in $x$ is claimed in Theorem 4.1 of 2 under some conditions, which are always satisfied if the equation is uniformly nondegenerate even if $c$ (see (2.2) ) is small. However, in this case, actually, the result of Theorem 4.1 of 2 is only proved for the derivatives along the mesh. This is rather harmless if the vectors on the mesh span the whole space, but excludes the cases when the mesh lies in a subspace, which happens, for instance, if we are dealing with, say, uniformly nondegenerate equations whose coefficients depend on a parameter and we want to estimate the the finite differences of their solutions with respect to the parameter by considering it as just another space variable. In that case no second-order derivatives with respect to the parameter enters the limit equation. The assumption that it is uniformly nondegenerate with respect to the original space variables does not help, and we need to have $c$ be large in order to rely on Theorem 4.1 of [2. Our results are free from this flaw; see Remarks 5.3 and 6.5 .

To understand faster the method and the results of the article, we advise the reader to concentrate only on the parabolic case and assume that the limit equation is uniformly nondegenerate. Then apart from the Lipschitz continuity, nothing else (see Remarks 2.1 and 2.3) is required for Theorem 2.1 to hold.

For the general equations our conditions (see Remarks 6.2 and 6.6) capture the main features of the corresponding conditions known from the theory of PDE. Namely, roughly speaking, we need the first-order derivatives in any direction along the mesh of the coefficients to be dominated either by the diffusion coefficients along the same direction or by $c$ or else by the drift term if it is sufficiently "monotone". It is worth noting that along the way we discover the necessity of the diffusion coefficients to have a special form and the usefulness of adding a diffusion term with a coefficient proportional to the mesh step into the approximating equation. This is similar to the method of artificial diffusion introduced in 9 .

Of course, in the same way as in 2, the results of the present article lead to the rate of convergence of order $h^{1 / 2}$ of approximating solutions to the true solution. However, for brevity we do not say more about this issue, only adding that in general our finite-difference equations need not be related in any way to a partial differential equation. 
Our main results are collected in Section 2, which also contains the proofs of all of them except Theorem 2.1. which is proved in Section 3 . In Section 2 we also state, in a special case, without proof one of the main results of the continuation of the present paper. Section 4 contains a discussion of our assumptions concerning the structure of the finite-difference equations under consideration. The point is that our equations do not contain mixed second-order differences and in Section 4 we explain that this is "almost" the most interesting case. The final Sections 5 and 6] are devoted to a rather long and detailed discussion of the somewhat formally stated Assumption 2.3 and show that it is natural in many cases alluded to above.

We are sincerely grateful to Hongjie Dong who read the article and made a few valuable comments. The comments made by the referee are also greatly appreciated.

\section{Formulation of the main RESUlts}

We take some numbers $h, T \in(0, \infty)$ and, in a cylindrical domain, consider the integral equation

$$
u(t, x)=g(x)+\int_{0}^{t}(L u(s, x)+f(s, x)) d s
$$

for $u$, where $g(x)$ and $f(s, x)$ are given real-valued Borel functions of $x=\left(x_{1}, \ldots, x_{d}\right)$ $\in \mathbb{R}^{d}$ and $(s, x) \in H_{T}:=[0, T] \times \mathbb{R}^{d}$, respectively, and $L$ is a linear operator given by

$$
\begin{aligned}
& L \varphi(t, x)=L_{h} \varphi(t, x)=L^{0} \varphi(t, x)-c(t, x) \varphi(x), \\
& L^{0} \varphi(t, x)=L_{h}^{0} \varphi(t, x)=\frac{1}{h} \sum_{\lambda \in \Lambda_{1}} q_{\lambda}(t, x) \delta_{\lambda} \varphi(x)+\sum_{\lambda \in \Lambda_{1}} p_{\lambda}(t, x) \delta_{\lambda} \varphi(x),
\end{aligned}
$$

for functions $\varphi$ on $\mathbb{R}^{d}$. Here $\Lambda_{1}$ is a finite subset of $\mathbb{R}^{d}$ such that $0 \notin \Lambda_{1}, p_{\lambda}(t, x)$, $q_{\lambda}(t, x)$ are real-valued functions of $(t, x) \in H_{T}$ given for each $\lambda \in \Lambda_{1}$, and

$$
\delta_{\lambda} \varphi(x)=\delta_{h, \lambda} \varphi(x)=\frac{1}{h}(\varphi(x+h \lambda)-\varphi(x)), \quad \lambda \in \Lambda_{1} .
$$

Let $m \geq 0$ be an integer and let $K_{1} \in[1, \infty)$ be a constant. Introduce

$$
\chi_{\lambda}=\chi_{h, \lambda}:=q_{\lambda}+h p_{\lambda} .
$$

We make the following assumptions.

Assumption 2.1. The functions $p, q, c, f$, and $g$ and their derivatives in $x$ up to order $m$ are bounded on $H_{T}$ and continuous in $x$.

Assumption 2.2. For all $(t, x) \in H_{T}$ and $\lambda \in \Lambda_{1}$,

$$
\chi_{\lambda}(t, x) \geq 0 \text {. }
$$

There exists a constant $c_{0}>0$ such that $c \geq c_{0}$.

Remark 2.1. The above assumption: $c \geq c_{0}>0$, is almost irrelevant if we only consider (2.1) on a finite time interval. Indeed, if $c$ is just bounded, say $|c| \leq$ $C=$ const, by introducing a new function $v(t, x)=u(t, x) e^{-2 C t}$ we will have an equation for $v$ similar to (2.1) with $L^{0} v-(c+2 C) v$ and $f e^{-2 C t}$ in place of $L u$ and $f$, respectively. Now for the new $c$ we have $c+2 C \geq C$. 
Remark 2.2. Introduce the following symmetry condition: (S) We have $\Lambda_{1}=-\Lambda_{1}$ and $q_{\lambda}=q_{-\lambda}$ on $\Lambda_{1}$.

Obviously under condition (S) we have

$$
h^{-1} \sum_{\lambda \in \Lambda_{1}} q_{\lambda}(t, x) \delta_{\lambda} \varphi(x)=(1 / 2) \sum_{\lambda \in \Lambda_{1}} q_{\lambda}(t, x) \Delta_{\lambda} \varphi(x),
$$

where

$$
\Delta_{\lambda} \varphi(x)=h^{-2}(\varphi(x+h \lambda)-2 \varphi(x)+\varphi(x-h \lambda)) .
$$

Take a function $\tau_{\lambda}$ defined on $\Lambda_{1}$ taking values in $[0, \infty)$, and for $\lambda \in \Lambda_{1}$ introduce the operators

$$
T_{\lambda} \varphi(x)=T_{h, \lambda} \varphi(x)=\varphi(x+h \lambda), \quad \bar{\delta}_{\lambda}=\tau_{\lambda} h^{-1}\left(T_{\lambda}-1\right) .
$$

It is worth noticing that in most applications we take $\tau_{\lambda} \equiv 1$ on $\Lambda_{1}$. However, there are cases (see Remark 5.3) in which it is useful to have some flexibility in changing $\tau_{\lambda}$.

For uniformity of notation we also introduce $\Lambda_{2}$ as the set of fixed distinct vectors $\ell^{1}, \ldots, \ell^{d}$ none of which is in $\Lambda_{1}$ and define

$$
\bar{\delta}_{\ell^{i}}=\bar{\delta}_{h, \ell^{i}}=\tau_{0} D_{i}:=\tau_{0} \partial / \partial x_{i}, \quad T_{\ell^{i}}=1, \quad \Lambda=\Lambda_{1} \cup \Lambda_{2},
$$

where $\tau_{0} \in[0,1]$ is a fixed constant. Observe that we allow $\tau_{0}$ to be zero in order to cover some results from 2 .

For $\mu \in \Lambda$ we set

$$
\begin{aligned}
Q \varphi & =h^{-1} \sum_{\lambda \in \Lambda_{1}} q_{\lambda} \delta_{\lambda} \varphi, \quad Q_{\mu} \varphi=h^{-1} \sum_{\lambda \in \Lambda_{1}}\left(\bar{\delta}_{\mu} q_{\lambda}\right) \delta_{\lambda} \varphi, \\
P \varphi & =\sum_{\lambda \in \Lambda_{1}} p_{\lambda} \delta_{\lambda} \varphi, \quad P_{\mu} \varphi=\sum_{\lambda \in \Lambda_{1}}\left(\bar{\delta}_{\mu} p_{\lambda}\right) \delta_{\lambda} \varphi, \\
L_{\mu}^{0} & =Q_{\mu}+P_{\mu} .
\end{aligned}
$$

Below, $B\left(\mathbb{R}^{d}\right)$ is the set of bounded Borel functions on $\mathbb{R}^{d}$ and $\mathfrak{K}$ is the set of bounded operators $\mathcal{K}=\mathcal{K}(t)$ mapping $B\left(\mathbb{R}^{d}\right)$ into itself preserving the cone of nonnegative functions and satisfying $\mathcal{K} 1 \leq 1$. We will often make use of the simple fact that for any $\mathcal{K}_{1}, \mathcal{K}_{2} \in \mathfrak{K}$ and nonnegative functions $\alpha, \beta$ on $\mathbb{R}^{d}$,

$$
\alpha \mathcal{K}_{1}+\beta \mathcal{K}_{2}=(\alpha+\beta) \mathcal{K}_{3}
$$

with

$$
\mathcal{K}_{3}:=\frac{\alpha}{\alpha+\beta} \mathcal{K}_{1}+\frac{\beta}{\alpha+\beta} \mathcal{K}_{2} \in \mathfrak{K} \quad\left(\frac{0}{0}:=0\right) .
$$

Assumption 2.3. We have $m \geq 1$ and there exist a constant $\delta \in(0,1]$ and an operator $\mathcal{K}=\mathcal{K}_{h}(t) \in \mathfrak{K}$, such that

$$
2 \sum_{\lambda \in \Lambda}\left(\bar{\delta}_{\lambda} \varphi\right) L_{\lambda}^{0} T_{\lambda} \varphi \leq \sum_{\lambda \in \Lambda} \mathcal{Q}\left(\bar{\delta}_{\lambda} \varphi\right)+K_{1} \mathcal{Q}(\varphi)+2(1-\delta) c \mathcal{K}\left(\sum_{\lambda \in \Lambda}\left|\bar{\delta}_{\lambda} \varphi\right|^{2}\right)
$$

on $H_{T}$ for all smooth functions $\varphi$, where

$$
\mathcal{Q}(\varphi)=\sum_{\mu \in \Lambda_{1}} \chi_{\mu}\left|\delta_{\mu} \varphi\right|^{2} .
$$

It is worth noting that Assumption 2.3 is automatically satisfied if $q_{\lambda}$ and $p_{\lambda}$ are independent of $x$. There are a few more cases when it is satisfied as well. We discuss some of them here and in Section 5 only mentioning right away three situations. 
Remark 2.3. Let Assumptions 2.1 and 2.2 hold with $m \geq 1$. Assume that $\Lambda_{1}=$ $-\Lambda_{1}, D q_{-\lambda}=D q_{\lambda}$ and $q_{\lambda} \geq \kappa$ for all $\lambda \in \Lambda_{1}$, where $\kappa>0$ is some constant. Then Assumption 2.3 is satisfied as well with $\delta$ as close to 1 as we wish, with $\tau_{\lambda} \equiv 1$ on $\Lambda_{1}$, appropriate $\tau_{0}>0, K_{1}$, unit $\mathcal{K}$, and all small $h$.

We will prove this remark at the end of this section. In Remark 6.4 we show that if we have $m \geq 2$ and the symmetry condition (S) is satisfied, then in the above remark the condition $\kappa>0$ can be replaced with $\kappa=0$, provided that $c_{0}$ is large enough (this time we need not assume that $h$ is small). In Remark 6.4 we also show that the condition $m \geq 2$ can be replaced with $m=1$ provided that $\sqrt{q_{\lambda}}$ are Lipschitz continuous in $x$ with a constant independent of $t$. In that case again Assumption 2.3 is satisfied for $\delta=1 / 10$ and appropriate $K_{1}, \tau_{0}>0$, provided that $c_{0}$ is large enough.

As we have seen in Remark 2.1, the condition that $c_{0}$ be large is, actually, harmless as long as we are concerned with equations on a finite time interval.

Fix a domain $Q \subset \mathbb{R}^{d}$ and introduce

$$
\begin{aligned}
& Q^{o}=\left\{x \in Q: x+\lambda h \in Q \quad \forall \lambda \in \Lambda_{1}\right\}, \quad \delta Q=Q \backslash Q^{o}, \\
& Q_{T}=[0, T] \times Q, \quad Q_{T}^{0}=[0, T] \times Q^{0}, \quad \delta_{x} Q_{T}=[0, T] \times \delta Q, \\
& \delta^{\prime} Q_{T}=(\{0\} \times Q) \cup \delta_{x} Q_{T} .
\end{aligned}
$$

Our first main result is formulated as follows, where by $D u$ we mean the gradient of $u$ with respect to $x$. Observe that the main case that $Q=\mathbb{R}^{d}$ is not excluded and in this case assumption (ii) of Theorem 2.1 below can be checked on the basis of Theorem 2.3. A typical and the most reasonable application of Theorem 2.1 when $Q$ is a proper domain is the case that $\tau_{0}=0$.

Theorem 2.1. (i) Let Assumptions 2.1 through 2.3 be satisfied and let $u$ be a bounded function on $H_{T}$ satisfying (2.1) in $Q_{T}$.

(ii) Assume that $u$ and Du are bounded and continuous in $Q_{T}$.

Then in $Q_{T}$ we have

$$
|u|+\tau_{0}|D u|+U \leq N\left(F_{1}+\sup _{\delta^{\prime} Q_{T}}\left(|u|+\tau_{0}|D u|+U\right)\right),
$$

where

$$
U=\left(\sum_{\lambda \in \Lambda_{1}}\left|\bar{\delta}_{\lambda} u\right|^{2}\right)^{1 / 2}, \quad F_{1}=\sup _{H_{T}}(|f|+|D f|),
$$

and $N$ depends only on $\delta, c_{0}, K_{1}, \sup _{H_{T}}|D c|$, and

$$
\left|\Lambda_{1}\right|^{2}:=\sum_{\lambda \in \Lambda_{1}}\left|\tau_{\lambda} \lambda\right|^{2} .
$$

Remark 2.4. We will see from the proof that, if $\tau_{0}=0$, then Theorem 2.1 holds without the assumption that $D u$ exists, let alone be continuous.

In case $Q=\mathbb{R}^{d}$, assumption (ii) of the following result is often satisfied due to Theorem 2.3 .

It is worth noting that if $Q=\mathbb{R}^{d}$, then $\delta Q=\emptyset$ and for any function $\varphi$ we set

$$
\sup _{\emptyset} \varphi:=0 \text {. }
$$


Theorem 2.2. (i) Let Assumptions 2.1$]$ through 2.3 be satisfied. Suppose that $q_{\lambda}$, $p_{\lambda}, c$, and $f$ are independent of $t$.

(ii) Assume that in $\mathbb{R}^{d}$ there exists a bounded function $u=u(x)$ which is bounded and continuous in $Q$ along with $D u$ and such that

$$
L u+f=0 \quad \text { in } Q \text {. }
$$

Then in $Q$ we have

$$
|u|+\tau_{0}|D u|+U \leq N\left(F_{1}+\sup _{\delta Q}\left(|u|+\tau_{0}|D u|+U\right),\right.
$$

where $U$ is the same as in Theorem 2.1 ,

$$
F_{1}=\sup _{\mathbb{R}^{d}}(|f|+|D f|),
$$

and $N$ depends only on $\delta, c_{0}, K_{1}, \sup _{\mathbb{R}^{d}}|D c|$, and $\left|\Lambda_{1}\right|$.

Proof. Take a sufficiently small constant $\nu>0$ so that $c-\nu \geq c_{0} / 2$ and Assumption 2.3 holds with $c-\nu$ and $\delta / 2$ in place of $c$ and $\delta$, respectively. Then observe that in $Q_{T}$ the function $v(t, x):=u(x) e^{\nu t}$ satisfies

$$
\frac{\partial}{\partial t} v=L^{0} v-(c-\nu) v+e^{\nu t} f
$$

and by Theorem 2.1 for $x \in Q$ and the obvious meaning of $V$ we have

$$
\begin{aligned}
& e^{\nu T}\left(|u(x)|+\tau_{0}|D u(x)|+U(x)\right)=|v(T, x)|+\tau_{0}|D v(T, x)|+V(T, x) \\
& \quad \leq N e^{\nu T}\left[F_{1}+\sup _{\delta Q}\left(|u|+\tau_{0}|D u|+U\right)\right]+N \sup _{Q}\left(|v|+\tau_{0}|D v|+V\right)(0, y) .
\end{aligned}
$$

By multiplying the extreme terms by $e^{-\nu T}$ and letting $T \rightarrow \infty$, we get the result. The theorem is proved.

Remark 2.5. It is worth noticing that in the above theorems it suffices that (2.3) be satisfied only in $Q_{T}^{o}$.

Theorem 2.3. (i) Let Assumption 2.1 be satisfied. Then there exists a unique bounded solution $u$ of (2.1) in $H_{T}$. Moreover, all derivatives in $x$ of $u$ of order $\leq m$ are bounded and continuous in $H_{T}$.

(ii) Let Assumptions 2.1 through 2.3 be satisfied. Suppose that $q_{\lambda}, p_{\lambda}, c$, and $f$ are independent of $t$. Then there exists a unique bounded solution $u=u(x)$ of the equation

$$
L u+f=0 \quad \text { in } \quad \mathbb{R}^{d} .
$$

Moreover, $u$ and Du are bounded and continuous in $\mathbb{R}^{d}$.

Proof. (i) Let $C^{m}$ be the space of functions on $\mathbb{R}^{d}$ which are bounded and continuous along with all derivatives up to order $m$. We endow $C^{m}$ with an appropriate sup norm and in the so-obtained Banach space, denoted again by $C^{m}$, consider the equation

$$
u(t)=g+\int_{0}^{t}(A(s) u(s)+f(s)) d s,
$$

where $f(s)=f(s, \cdot)$ and $A(s)$ are operators in $C^{m}$ given by

$$
A(s) \varphi(x)=h^{-1} \sum_{\lambda \in \Lambda_{1}} \chi_{\lambda}(s, x) \delta_{\lambda} \varphi(x)-c(s, x) \varphi(x) .
$$


Owing to Assumption 2.1.

$$
\|A(s) \varphi\|_{C^{m}} \leq N\|\varphi\|_{C^{m}}
$$

with $N$ independent of $s$ and $\varphi$. Hence, our result is a direct consequence of the general theorem about ordinary differential equations in Banach spaces (for proving uniqueness we take $m=0$ ).

(ii) By assertion (i) for any $T$ there exists a unique bounded and continuous in $H_{T}$ solution $v(t, x)$ of the problem

$$
\frac{\partial}{\partial t} v(t, x)=(L+\nu) v(t, x) \quad t>0, \quad v(0, x)=f(x),
$$

where $\nu=c_{0} / 2$. In addition, $D v$ is bounded and continuous in $H_{T}$ for each $T$. By Theorem 2.1, $v$ and $D v$ are bounded and continuous in $H_{\infty}$. Define

$$
u(x)=\int_{0}^{\infty} e^{-\nu t} v(t, x) d t .
$$

Then the rules of differentiating under the integral sign and the dominated convergence theorem show that $u$ and $D u$ are bounded and continuous. Furthermore, integrating by parts, we see that

$$
L u(x)=\int_{0}^{\infty} e^{-\nu t} L v(t, x) d t=\int_{0}^{\infty} e^{-\nu t}\left[\frac{\partial}{\partial t} v(t, x)-\nu v(t, x)\right] d t=-f(x),
$$

so that $u$ satisfies (2.6).

To prove the uniqueness of bounded solutions of (2.6) we use Lemma 3.1, which is proved in Section 3. If $w$ is the difference of two bounded solutions of (2.6), then $v(t, x):=w(x) e^{c_{0} t}$ satisfies (2.5) with $\nu=c_{0}$ and $f=0$. Since $c-\nu \geq 0$, by Lemma 3.1 .

$$
v(t, x) \leq \sup _{\mathbb{R}^{d}} v_{+}(0, y)=\sup _{\mathbb{R}^{d}} w_{+}, \quad w(x) \leq e^{-c_{0} t} \sup _{\mathbb{R}^{d}} w_{+}
$$

and by letting $t \rightarrow \infty$ we obtain $w \leq 0$. The same inequality holds for $-w$, so that $w=0$, which proves uniqueness and finishes the proof of the theorem.

Remark 2.6. Let Assumption 2.1 hold. Then it is easy to see that for the bounded solution $u$ of (2.1) in $H_{T}$ for each fixed $h_{1}>0$,

$$
\sup _{(t, x) \in H_{T}}|u(t, x)| \leq N\left(\sup _{(t, x) \in H_{T}}|f(t, x)|+\sup _{x \in \mathbb{R}^{d}}|g(x)|\right)
$$

holds for all $h>h_{1}$, where $N$ is a constant independent of $h$.

Remark 2.7. A simple inspection of their proof shows that the above theorems remain valid if $\mathcal{K}\left(\sum_{\lambda \in \Lambda}\left|\bar{\delta}_{\lambda} \varphi\right|^{2}\right)$ in Assumption 2.3 is replaced by

$$
\sup _{x} \sum_{\lambda \in \Lambda}\left|\bar{\delta}_{\lambda} \varphi\right|^{2} .
$$

We do not know how much can be gained by such weakening of Assumption 2.3. On the other hand, in a subsequent article we will see an advantage of using operators $\mathcal{K} \in \mathfrak{K}$.

Now we state without proof one of the main results of the forthcoming paper [5]. As we know from Remark 2.3 and Theorem 2.3 (ii), under the conditions of Theorem 2.4 (see below), for each $h>0$, there exists a unique bounded solution $u_{h}$ of

$$
L_{h} u+f=0 \quad \text { in } \mathbb{R}^{d} .
$$


For a fixed integer $k \geq 0$ set

$$
v_{h}=\sum_{j=0}^{k} b_{j} u_{2^{-j} h},
$$

where

$$
\left(b_{0}, b_{1}, \ldots, b_{k}\right):=(1,0,0, \ldots, 0) V^{-1}
$$

and $V^{-1}$ is the inverse of the Vandermonde matrix with entries

$$
V^{i j}:=2^{-(i-1)(j-1)}, \quad i, j=1, \ldots, k+1 .
$$

Consider also the equation

$$
\mathcal{L} v+f=0 \quad \text { in } \quad \mathbb{R}^{d}
$$

with

$$
\begin{aligned}
& \mathcal{L}:=a_{i j} D_{i} D_{j}+b_{i} D_{i}-c, \\
& a_{i j}(x):=(1 / 2) \sum_{\lambda \in \Lambda_{1}} q_{\lambda}(x) \lambda_{i} \lambda_{j}, \quad b_{i}:=\sum_{\lambda \in \Lambda_{1}} p_{\lambda}(x) \lambda_{i} .
\end{aligned}
$$

One of our main theorems from [5] in a special case reads as follows.

Theorem 2.4. Let $m \geq 3(k+1)$ for some integer $k \geq 0$. Let Assumptions 2.1, 2.2. and the symmetry assumption $(S)$ be satisfied. Also assume that $q_{\lambda}(x) \geq \kappa$ for all $\lambda \in \Lambda_{1}, x \in \mathbb{R}^{d}$ for some constant $\kappa>0$.

Then there is a unique bounded solution $v$ to (2.7) and

$$
\left|v_{h}(x)-v(x)\right| \leq N h^{k+1}
$$

for all $x \in \mathbb{R}^{d}, h \in\left(0, h_{0}\right]$, and every $h_{0}>0$, where $N$ is a constant depending only on $h_{0}, k, m, \kappa, c_{0},\left|\Lambda_{1}\right|$, and on the sup norms of the derivatives of $q_{\lambda}, p_{\lambda}, c$, and $f$ up to order $m$.

We obtain this result in [5] by showing that the derivatives of $v_{h}$ in $h$ up to order $k+1$ are bounded functions of $h \in\left(0, h_{0}\right]$, which we will prove via our estimates on the derivatives of $v_{h}$ in $x$. The reader may wonder why we do not choose the straightforward way of estimating the derivatives of $v_{h}$ in $h$ via an "explicit" formula for $v$. To test this approach we suggest the reader try to estimate $d v_{h} / d h$ directly for

$$
v_{h}(x)=\frac{h^{2}}{2+h^{2}} f(x)+\sum_{n=1}^{\infty} h^{2}\left(\frac{2}{2+h^{2}}\right)^{n+1} E f\left(x+\sum_{i=1}^{n} h \varepsilon_{i}\right),
$$

where $\varepsilon_{i}$ are independent random variables taking 1 and -1 with probability $1 / 2$, without noticing that $v_{h}$ is the bounded solution of

$$
\frac{1}{h^{2}}(u(x+h)-2 u(x)+u(x-h))-u(x)+f(x)=0, \quad x \in \mathbb{R} .
$$

We finish the section by proving the assertion in Remark 2.3. Clearly,

$$
2 \sum_{\lambda \in \Lambda}\left(\bar{\delta}_{\lambda} \varphi\right) L_{\lambda}^{0} T_{\lambda} \varphi=I_{1}+I_{2}
$$

with

$$
I_{1}:=2 \sum_{\lambda \in \Lambda_{1}}\left(\bar{\delta}_{\lambda} \varphi\right) L_{\lambda}^{0} T_{\lambda} \varphi, \quad I_{2}:=2 \sum_{\lambda \in \Lambda_{2}}\left(\bar{\delta}_{\lambda} \varphi\right) L_{\lambda}^{0} \varphi
$$


We take $\tau_{\lambda} \equiv 1$ on $\Lambda_{1}$ and notice that, due to the symmetry of $\Lambda_{1}$ and the symmetry of $D q_{\lambda}$ in $\lambda$,

$$
\begin{aligned}
I_{1}= & 2 \sum_{\lambda \in \Lambda_{1}}\left(\delta_{\lambda} \varphi\right) L_{\lambda}^{0} \varphi+2 h \sum_{\lambda \in \Lambda_{1}}\left(\delta_{\lambda} \varphi\right) L_{\lambda}^{0} \delta_{\lambda} \varphi \\
= & \sum_{\lambda, \mu \in \Lambda_{1}}\left(\delta_{\lambda} \varphi\right)\left(\delta_{\lambda} q_{\mu}\right) \Delta_{\mu} \varphi+2 \sum_{\lambda, \mu \in \Lambda_{1}}\left(\delta_{\lambda} \varphi\right)\left(\delta_{\lambda} p_{\mu}\right) \delta_{\mu} \varphi \\
& +2 \sum_{\lambda, \mu \in \Lambda_{1}}\left(\delta_{\lambda} \varphi\right)\left(\delta_{\lambda} \chi_{\mu}\right) \delta_{\mu} \delta_{\lambda} \varphi=: I_{1}^{(1)}+I_{1}^{(2)}+I_{1}^{(3)}, \\
I_{2}= & I_{2}^{(1)}+I_{2}^{(2)},
\end{aligned}
$$

where in the notation $\xi=D \varphi /|D \varphi|, \psi_{(\xi)}=\xi_{i} D_{i} \psi$,

$$
\begin{aligned}
I_{2}^{(1)} & =\tau_{0}^{2} \sum_{j=1}^{d} \sum_{\mu \in \Lambda_{1}}\left(D_{j} \varphi\right)\left(D_{j} q_{\mu}\right) \Delta_{\mu} \varphi=\tau_{0}^{2}|D \varphi| \sum_{\mu \in \Lambda_{1}} q_{\mu(\xi)} \Delta_{\mu} \varphi \\
I_{2}^{(2)} & =2 \tau_{0}^{2} \sum_{j=1}^{d} \sum_{\mu \in \Lambda_{1}}\left(D_{j} \varphi\right)\left(D_{j} p_{\mu}\right) \delta_{\mu} \varphi=2 \tau_{0}^{2}|D \varphi| \sum_{\mu \in \Lambda_{1}} p_{\mu(\xi)} \delta_{\mu} \varphi .
\end{aligned}
$$

By Young's inequality, taking into account that $\chi_{\lambda} \geq \kappa / 2>0$ for sufficiently small $h$, and that $c \geq c_{0}>0$, we have

$$
\begin{aligned}
I_{1}^{(j)} & \leq(1 / 3) \sum_{\lambda \in \Lambda_{1}} \mathcal{Q}\left(\delta_{\lambda} \varphi\right)+N \mathcal{Q}(\varphi) \quad \text { for } j=1,3, \quad I_{1}^{(2)} \leq N \mathcal{Q}(\varphi), \\
I_{2}^{(1)} & \leq(1 / 3) \sum_{\mu \in \Lambda_{1}} \mathcal{Q}\left(\delta_{\mu} \varphi\right)+\tau_{0}^{2} N c_{0}^{-1} c \sum_{\lambda \in \Lambda_{2}}\left|\bar{\delta}_{\lambda} \varphi\right|^{2}, \\
I_{2}^{(2)} & \leq \tau_{0} N c_{0}^{-1} c \sum_{\lambda \in \Lambda}\left|\bar{\delta}_{\lambda} \varphi\right|^{2},
\end{aligned}
$$

where $N$ is a constant depending only on $\kappa$, the number of elements in $\Lambda_{1}$ and on the supremum norm of the gradients of $p_{\lambda}$ and $q_{\lambda}$ in $x$. Summing up these inequalities and taking $\tau_{0}>0$ sufficiently small we get (2.3) with $K_{1}=3 N$, unit operator $\mathcal{K}$, and with $\delta$ as close to 1 as we wish.

\section{Proof of Theorem 2.1}

If $Q=\mathbb{R}^{d}$, Theorem 2.3 (i) shows that equation (2.1) has a unique bounded continuous solution $u$ for which the partial derivatives in $x \in \mathbb{R}^{d}$ up to order $m$ are bounded continuous functions of $(t, x)$. However, the bounds, which can be extracted from the proof of Theorem 2.3 for these derivatives depend on the parameters $h$ and $T$. Our aim is to show the existence of bounds, independent of $h$ and $T$ if $m=1$ and in addition to Assumption 2.1. Assumptions 2.2 and 2.3 also hold. We will obtain such estimates by making use of the following version of the maximum principle. It is probably worth drawing the reader's attention to the fact that the assumption that $c$ has a certain sign is not used in Lemma 3.1

Lemma 3.1. Let Assumption 2.1 with $m=0$ be satisfied and let $\chi_{\lambda} \geq 0$ for all $\lambda \in \Lambda_{1}$. Let $v$ be a bounded function on $Q_{T}$, such that $v(\cdot, x)$ is measurable for any $x \in Q$ and the partial derivative $D_{t} v:=\partial v(t, x) / \partial t$ exists in $Q_{T}^{o}$. Let $F(t) \geq 0$ be 
an integrable function on $[0, T]$, and let $C(t, x) \geq 0$ be a bounded function. Assume that for all $(t, x) \in Q_{T}^{o}$ we have

$$
D_{t} v \leq L v+C \bar{v}_{+}+F,
$$

where $\bar{v}(t)=\sup \{v(t, x): x \in Q\}$.

Then in $[0, T]$ we have

$$
\bar{v}(t) \leq G(t) e^{\nu t}+\int_{0}^{t} F(s) e^{\nu(t-s)} d s,
$$

where

$$
\begin{aligned}
& \nu:=\sup _{Q_{T}^{o}}(C-c), \quad G(t)=\sup _{(s, y) \in \delta^{\prime} Q_{t}} e^{-\nu s} v_{+}(s, y), \\
& \delta^{\prime} Q_{t}=(\{0\} \times Q) \cup \delta_{x} Q_{t} .
\end{aligned}
$$

Proof. First assume that $C=c=F=G=0$. In that case introduce $\tilde{v}(t, x)=$ $v(t, x) e^{N t}$, where

$$
N=N_{h}=\sup _{Q_{T}} h^{-1} \sum_{\lambda \in \Lambda_{1}} \chi_{\lambda} .
$$

Observe that $v(t, x)=\tilde{v}(t, x) e^{-N t}$,

$$
\begin{gathered}
e^{-N t}\left(D_{t} \tilde{v}(t, x)-N \tilde{v}(t, x)\right) \leq e^{-N t} L \tilde{v}(t, x), \\
D_{t} \tilde{v}(t, x) \leq(L+N) \tilde{v}(t, x)
\end{gathered}
$$

in $Q_{T}^{0}$, and in $Q_{T}$,

$$
\tilde{v}(t, x) \leq J \tilde{v}(t, x):=I_{Q_{T}^{0}} \int_{0}^{t}(L+N)\left(I_{Q_{T}} \tilde{v}\right)(s, x) d s .
$$

Obviously, due to the choice of $N$ and the assumption that $\chi_{\lambda} \geq 0$, if $u_{1} \geq u_{2}$ on $Q_{T}$, then $J u_{1} \geq J u_{2}$ on $Q_{T}$. It follows that $\tilde{v} \leq J^{k} \tilde{v}$ for any $k$.

Observe that for any bounded function $f$,

$$
\sup _{Q}|J f(t, \cdot)| \leq N^{\prime} \int_{0}^{t} \sup _{Q}|f(s, \cdot)| d s,
$$

where $N^{\prime}$ is independent of $f$ and $t$. It follows easily that for any bounded function $f$ we have $J^{k} f \rightarrow 0$ uniformly on $Q_{T}$. Hence $\tilde{v} \leq 0$ and $v \leq 0$ in $Q_{T}$.

Now we consider another particular case in which $G=c=0$ and $C=$ const $\geq 0$. Then observe that the function

$$
w(t, x)=\int_{0}^{t}\left(C \bar{v}_{+}+F\right)(s) d s
$$

satisfies

$$
D_{t} w=L w+C \bar{v}_{+}+F .
$$

Therefore $\hat{v}:=v-w$ satisfies $D_{t} \hat{v} \leq L \hat{v}$. In addition, $\hat{v} \leq 0$ on $\delta^{\prime} Q_{T}$. By the above, on $Q_{T}$ we have $\hat{v} \leq 0$, that is,

$$
v(t, x) \leq \int_{0}^{t}\left(C \bar{v}_{+}+F\right)(s) d s, \quad \bar{v}_{+}(t) \leq \int_{0}^{t}\left(C \bar{v}_{+}+F\right)(s) d s,
$$

and (3.2) follows by Gronwall's inequality and the fact that $\nu=C$. 
Now we allow $c \neq 0$ and variable $C$, but still assume that $G=0$. In that case take a large constant $M$ so that $M>c$ and $M+\nu>0$ and for $\hat{v}(t, x)=v(t, x) e^{M t}$ write

$$
e^{-M t}\left(D_{t} \hat{v}(t, x)-M \hat{v}(t, x)\right) \leq e^{-M t} L \hat{v}(t, x)+C(t, x) \bar{v}_{+}(t)+F(t) .
$$

Dropping obvious values of the arguments and introducing

$$
\overline{\hat{v}}(t)=\sup _{x \in Q} \hat{v}(t, x) \quad\left(=\bar{v} e^{M t}\right),
$$

we find

$$
\begin{aligned}
& D_{t} \hat{v} \leq L^{0} \hat{v}+(M-c) \hat{v}+C \overline{\hat{v}}_{+}+e^{M t} F \leq L^{0} \hat{v}+(M-c) \overline{\hat{v}}_{+} \\
& \quad+C \overline{\hat{v}}_{+}+e^{M t} F \leq L^{0} \hat{v}+(M+\nu) \overline{\hat{v}}_{+}+e^{M t} F .
\end{aligned}
$$

It follows by the above that

$$
\bar{v}(t) e^{M t} \leq \int_{0}^{t} e^{M s} F(s) e^{(M+\nu)(t-s)} d s,
$$

which is equivalent to (3.2). By the way, notice that so far we have not used the fact that $C \geq 0$.

Now comes the general case in which we set

$$
w(t, x)=v(t, x) e^{-\nu t}-\beta, \quad \beta=\sup _{(s, y) \in \delta^{\prime} Q_{T}} v_{+}(s, y) e^{-\nu s} .
$$

Simple manipulations show that (3.1) becomes

$$
D_{t} w \leq L^{0} w-c(w+\beta)-\nu(w+\beta)+C e^{-\nu t} \bar{v}_{+}+F e^{-\nu t},
$$

where

$$
e^{-\nu t} \bar{v}_{+} \leq \bar{w}_{+}+\beta, \quad \bar{w}_{+}(t):=\sup _{Q} w_{+}(t, x)
$$

and, owing to the assumption that $C \geq 0$, the definition of $\nu$, and the fact that $\beta \geq 0$,

$$
C e^{-\nu t} \bar{v}_{+} \leq C \bar{w}_{+}+C \beta \leq C \bar{w}_{+}+(c+\nu) \beta .
$$

It follows that

$$
D_{t} w \leq L^{0} w-(c+\nu) w+C \bar{w}_{+}+F e^{-\nu t} .
$$

Since $w \leq 0$ on $\delta^{\prime} Q_{T}$ and $\sup (C-(c+\nu))=0$, by the above cases we have for $t \leq T$ that

$$
\begin{gathered}
\bar{w}(t) \leq \int_{0}^{t} F(s) e^{-\nu s} d s \\
\bar{v}(t) \leq e^{\nu t} \sup _{(s, y) \in \delta^{\prime} Q_{T}} v_{+}(s, x) e^{-\nu s}+e^{\nu t} \int_{0}^{t} F(s) e^{-\nu s} d s .
\end{gathered}
$$

We can put here $t=T$ and then, by using certain freedoms in choosing the end of the time interval, we can, actually, set $T=t$ in (3.3). Then we arrive at (3.2) for all $t \leq T$. The lemma is proved. 
Corollary 3.2. Under the conditions of Lemma 3.1, if $\nu<0$, then

$$
\bar{v}(t) \leq \sup _{(s, y) \in \delta^{\prime} Q_{t}} v_{+}(s, y)+|\nu|^{-1} \sup _{[0, t]} F .
$$

Proof of Theorem 2.1. Introduce

$$
V_{0}=u^{2}, \quad V_{1}=\sum_{\lambda \in \Lambda}\left|\bar{\delta}_{\lambda} u\right|^{2}, \quad \bar{V}_{k}(t)=\sup _{x \in Q} V_{k}(t, x),
$$

recall that $F_{1}$ is introduced in the statement of Theorem 2.1 and set

$$
G=\sup _{\delta^{\prime} Q_{T}}\left(|u|+\tau_{0}|D u|+U\right) .
$$

By Corollary 3.2 applied to $u$ or $-u$ from the assumption that $c \geq c_{0}$ we obtain that

$$
V_{0}=|u|^{2} \leq\left(G+c_{0}^{-1} F_{1}\right)^{2} .
$$

Now we use the formula

$$
\delta_{\lambda}(\psi \varphi)=\left(\delta_{\lambda} \psi\right) T_{\lambda} \varphi+\psi \delta_{\lambda} \varphi=\left(\delta_{\lambda} \psi\right) \varphi+\psi \delta_{\lambda} \varphi+h\left(\delta_{\lambda} \psi\right) \delta_{\lambda} \varphi, \quad \lambda \in \Lambda_{1},
$$

to get

In particular,

$$
L^{0}\left(\varphi^{2}\right)=2 \varphi L^{0} \varphi+\mathcal{Q}(\varphi)
$$

$$
L^{0} V_{1}=2 \sum_{\lambda \in \Lambda}\left(\bar{\delta}_{\lambda} u\right) L^{0} \bar{\delta}_{\lambda} u+\sum_{\lambda \in \Lambda} \mathcal{Q}\left(\bar{\delta}_{\lambda} u\right)
$$

We observe that

$$
L^{0} \bar{\delta}_{\lambda} u=\bar{\delta}_{\lambda} L^{0} u-L_{\lambda}^{0} T_{\lambda} u
$$

if $\lambda \in \Lambda$, and in $Q_{T}^{o}$,

$$
\bar{\delta}_{\lambda} L^{0} u=D_{t} \bar{\delta}_{\lambda} u+c \bar{\delta}_{\lambda} u+\left(\bar{\delta}_{\lambda} c\right) T_{\lambda} u-\bar{\delta}_{\lambda} f .
$$

Then in $Q_{T}^{o}$ we find

$$
\begin{aligned}
& L^{0} V_{1}-D_{t} V_{1}-2 c V_{1}=\sum_{\lambda \in \Lambda} \mathcal{Q}\left(\bar{\delta}_{\lambda} u\right) \\
& \quad+2 \sum_{\lambda \in \Lambda}\left(\bar{\delta}_{\lambda} u\right)\left(\left(\bar{\delta}_{\lambda} c\right) T_{\lambda} u-\bar{\delta}_{\lambda} f\right)-2 \sum_{\lambda \in \Lambda}\left(\bar{\delta}_{\lambda} u\right) L_{\lambda}^{0} T_{\lambda} u .
\end{aligned}
$$

We use Assumption 2.3 to conclude that

$$
L^{0} V_{1}-D_{t} V_{1}-2 c V_{1} \geq-K_{1} \mathcal{Q}(u)-2(1-\delta) c \bar{V}_{1}+I,
$$

where

$$
I:=2 \sum_{\lambda \in \Lambda}\left(\bar{\delta}_{\lambda} u\right)\left(\left(\bar{\delta}_{\lambda} c\right) T_{\lambda} u-\bar{\delta}_{\lambda} f\right) .
$$

Notice that by Young's inequality,

$$
2 \sum_{\lambda \in \Lambda} \mid\left(\bar{\delta}_{\lambda} u\right)\left(\left(\bar{\delta}_{\lambda} c\right) T_{\lambda} u \mid \leq \delta^{2} \bar{V}_{1}+\delta^{-2} \bar{V}_{0} \sum_{\lambda \in \Lambda}\left(\bar{\delta}_{\lambda} c\right)^{2},\right.
$$

where, for each $\lambda \in \Lambda_{1}$,

$$
\begin{aligned}
& \delta_{\lambda} c(t, x)=h^{-1} \int_{0}^{h} \lambda_{i} D_{i} c(t, x+\lambda \theta) d \theta, \\
& \left|\bar{\delta}_{\lambda} c(t, x)\right|^{2} \leq\left|\tau_{\lambda} \lambda\right|^{2} h^{-1} \int_{0}^{h}|D c(t, x+\lambda \theta)|^{2} d \theta \leq\left|\tau_{\lambda} \lambda\right|^{2} \sup _{H_{T}}|D c|^{2},
\end{aligned}
$$


so that

$$
\begin{aligned}
& \sum_{\lambda \in \Lambda}\left(\bar{\delta}_{\lambda} c\right)^{2} \leq \sup _{H_{T}}|D c|^{2}\left(1+\left|\Lambda_{1}\right|^{2}\right) \\
& 2 \sum_{\lambda \in \Lambda}\left|\left(\bar{\delta}_{\lambda} u\right)\left(\bar{\delta}_{\lambda} c\right) T_{\lambda} u\right| \leq \delta^{2} \bar{V}_{1}+N \bar{V}_{0} \leq \delta^{2} \bar{V}_{1}+N\left(F_{1}^{2}+G^{2}\right) .
\end{aligned}
$$

Similarly,

$$
2 \sum_{\lambda \in \Lambda}\left|\left(\bar{\delta}_{\lambda} u\right) \bar{\delta}_{\lambda} f\right| \leq \delta^{2} \bar{V}_{1}+N F_{1}^{2}
$$

Hence (3.4) yields

$$
L^{0} V_{1}-D_{t} V_{1}-2 c V_{1} \geq-2\left(\delta^{2}+c-\delta c\right) \bar{V}_{1}-K_{1} \mathcal{Q}(u)-N\left(F_{1}^{2}+G^{2}\right) .
$$

Next,

$$
\begin{aligned}
& L^{0}\left(u^{2}\right)-2 c u^{2}-D_{t}\left(u^{2}\right)=2 u\left(L^{0} u-c u-D_{t} u\right)+\mathcal{Q}(u) \\
& \quad=-2 u f+\mathcal{Q}(u) \geq-N\left(F_{1}^{2}+G^{2}\right)+\mathcal{Q}(u) .
\end{aligned}
$$

This, (3.5), and the fact that $\delta^{2}+c-\delta c \geq 0$ show that for

$$
W:=V_{1}+K_{1} u^{2}, \quad \bar{W}=\sup _{Q} W(\cdot, x),
$$

we have

$$
L^{0} W-2 c W-D_{t} W \geq-2\left(\delta^{2}+c-\delta c\right) \bar{W}-N\left(F_{1}^{2}+G^{2}\right) .
$$

Now we want to use Corollary 3.2 , Set

$$
\nu:=\sup _{Q_{T}^{o}}\left[2\left(\delta^{2}+c-\delta c\right)-2 c\right]=2 \delta \sup _{Q_{T}^{o}}(\delta-c) \leq 2 \delta\left(\delta-c_{0}\right) .
$$

If

$$
\nu \leq-\delta c_{0},
$$

then by Corollary 3.2 we get $W \leq N\left(F_{1}^{2}+G^{2}\right)$, which obviously implies (2.4). Finally, observe that, if Assumption 2.3 is satisfied with a $\delta=\delta_{0}>0$, then it is also satisfied with any $\delta \in\left(0, \delta_{0}\right]$ and the same $K_{1}, \mathcal{K}$. Hence by modifying $\delta$ if necessary, so that $\delta \leq c_{0} / 2$, we satisfy (3.6), thus proving the theorem.

The following remark will be used in a subsequent paper when we will be estimating higher-order derivatives of $u$.

Remark 3.1. Suppose that, instead of Assumption 2.3. Assumption 5.1 (see Section 5) is satisfied. Then a simple inspection of the above proof shows that in place of (3.5) we have

$$
\begin{aligned}
& L^{0} V_{1}-D_{t} V_{1}-2 c V_{1} \geq-2\left(\delta^{2}+c-\delta c\right) \bar{V}_{1} \\
& \quad+\delta \sum_{\lambda \in \Lambda} \mathcal{Q}\left(\bar{\delta}_{\lambda} u\right)-K_{1} \mathcal{Q}(u)-N\left(F_{1}^{2}+G^{2}\right) .
\end{aligned}
$$




\section{Some issues Related to the COnVERGence $L_{h} \rightarrow \mathcal{L}$}

There is a natural question about the relation of the finite-difference operators $L=L_{h}$ with partial-differential operators. We certainly want to apply the results of the present article to investigating approximate solutions of elliptic and parabolic second-order equations. Then, given an elliptic operator

$$
\mathcal{L}=a_{i j} D_{i} D_{j}+b_{i} D_{i}
$$

with variable coefficients, a natural question arises as to whether it is possible to construct operators $L_{h}$ such that they converge to $\mathcal{L}$ and our assumptions are satisfied.

This question has little to do with the dependence of $a_{i j}$ and $b_{i}$ on $t$, and we assume that

$$
a_{i j}=a_{i j}(x), \quad b_{i}=b_{i}(x),
$$

and $a$ and $b$ are bounded and continuous along with their first-order derivatives.

It is not hard to see that under the symmetry assumption $(\mathrm{S})$, the operators $L_{h}$ approximate $\mathcal{L}$ with

$$
\begin{aligned}
& a_{i j}(x)=(1 / 2) \sum_{\lambda \in \Lambda_{1}} q_{\lambda}(x) \lambda_{i} \lambda_{j} \\
& b_{i}=\sum_{\lambda \in \Lambda_{1}} p_{\lambda}(x) \lambda_{i}
\end{aligned}
$$

in the sense that $L_{h} \varphi \rightarrow \mathcal{L} \varphi$ as $h \downarrow 0$ for all smooth $\varphi$.

A few basic examples describing conditions on $q_{\lambda}$, which guarantee that our assumptions are satisfied, are given in Remarks 2.3, 6.4, and 6.11. However, how this can be transformed into some conditions in terms of $a_{i j}$ is not clear right away.

By the way, it is shown in [1] that if $\mathcal{L}$ admits finite-difference approximations constructed by contracting a fixed mesh and the approximating operators satisfy the maximum principle, then they always have the form (2.2) with $\Lambda_{1}=-\Lambda_{1}$ and $q_{\lambda}=q_{-\lambda}$. This form is nonunique and the issue of choosing appropriate $q_{\lambda}$ and $p_{\lambda}$ arises.

It is proved in [7] that, if the matrix $a$ is uniformly nondegenerate, then there always exist $\Lambda_{1}$ and $q_{\lambda}$ possessing property (S), such that (4.1) holds, $q_{\lambda}$ are as smooth as $a$ is, and $q_{\lambda} \geq \kappa>0$, where $\kappa$ is a constant.

It is also proved in [7] that if all values of the matrix $a$ lie in a closed convex polyhedron in the set of nonnegative matrices and $a(x)$ has two bounded derivatives, then again there exist $\Lambda_{1}$ and $q_{\lambda}$ possessing property (S), such that (4.1) holds, and $\sqrt{q_{\lambda}}$ are Lipschitz continuous.

In these two cases the issue of satisfying our assumptions reduces to representing $b(x)$ appropriately.

There is a way to do so, used quite often in probabilistic literature, by adding, if necessary, the set $\Gamma=\left\{ \pm e^{1}, \ldots, \pm e^{d}\right\}$ to $\Lambda_{1}$, where $\left\{e^{i}\right\}$ is the standard basis in $\mathbb{R}^{d}$, defining

$$
p_{ \pm e^{i}}=\left(b_{i}\right)_{ \pm}
$$

$\left(t_{ \pm}=(1 / 2)(|t| \pm t)\right)$, and defining $p_{\lambda}=0$ on the remaining part of $\Gamma \cup \Lambda_{1}$. There is a certain inconvenience in this approximation, which we discuss in the following example along with a way to avoid it by using different $p_{\lambda}$ 's. 
Example 4.1. For $d=1$ consider the operator

$$
L u=b D u,
$$

where $b=b(x)$ is a smooth function bounded along with its derivatives.

If $b$ changes sign, then, since in our setting $\chi_{\lambda}=h p_{\lambda}$ is required to be $\geq 0$, we have to take $\Lambda_{1}$ consisting of at least two points $\left\{\lambda_{1}, \lambda_{3}\right\}$. The most natural choice is $\Lambda_{1}=\{ \pm 1\}$ and

$$
L_{h} \varphi(x)=P_{h} \varphi(x)=b_{+}(x) \delta_{h, 1} \varphi(x)+b_{-}(x) \delta_{h,-1} \varphi(x) .
$$

Observe that on smooth $\varphi$ we have $L_{h} \varphi \rightarrow b D \varphi$ as $h \downarrow 0$.

Notice that if $b$ changes sign, $p_{ \pm 1}=b_{ \pm}$are Lipschitz continuous but need not be continuously differentiable unless we impose a severe restriction on the behavior of $b$ near the points where it vanishes. Actually, in this article, the assumption that $q_{\lambda}$ and $p_{\lambda}$ are smooth can be replaced with the assumption that they are Lipschitz continuous and then require (2.3) to be satisfied for each $t$ almost everywhere with respect to $x$ rather than for all $x$. However, in such a case it is unrealistic to assume in Theorem 2.1 that $u$ is continuously differentiable in $x$. Generally, $u$ will be only Lipschitz continuous in $x$ and estimate (2.4) will hold almost everywhere rather than everywhere in $Q_{T}$. More serious trouble occurs when we want to estimate higher-order derivatives, which we will be concerned with in a subsequent article. Then we need $p_{\lambda}$ to have higher-order derivatives and this excludes many interesting cases.

On the other hand, the reader may like to check that, with the above $p$, condition (2.3) is satisfied (a.e.) with any $\delta \in(0,1)$ as long as $b$ is a decreasing function, which agrees well with the limit case of differential equations.

One can construct a different approximation of $b D \varphi$ for which $p_{\lambda}$ are as smooth as $b$. Indeed, take a constant $\theta$ such that $|b|+1 \leq \theta$ and set

$$
p_{1}=b+\theta, \quad p_{-1}=\theta .
$$

Then $p_{ \pm 1} \geq 1$, again $p_{1} \delta_{h, 1} \varphi+p_{-1} \delta_{h,-1} \varphi \rightarrow b D \varphi$ on smooth $\varphi$, and $p_{ \pm 1}$ are as smooth as $b$. This method is somewhat close to adding an artificial diffusion. However, we add it only to the finite-difference approximation and not to the operator $b D$.

Now consider the operator

$$
L u=(1 / 2) a D^{2}+b D u,
$$

where we suppose that $a(x) \geq 0$ and $r:=\sqrt{a}$ and $b$ are one time differentiable with derivatives uniformly continuous on $\mathbb{R}$. Again take $\Lambda_{1}=\{ \pm 1\}$ and construct $p_{\lambda}$ as in Remark 6.6 and define $q_{\lambda}=a$ and $r_{\lambda}=r=\sqrt{a}$.

It is shown in Remark 6.7 that for $h$ sufficiently small, Assumption 2.3 holds (perhaps with different $\delta$ and $K_{1}$ ) if

$$
14\left(r^{\prime}\right)^{2}+b^{\prime} \leq(1-\delta) c+K_{1} a .
$$

This condition describes what we need from $\mathcal{L}$ in the one-dimensional case and it looks quite satisfactory. On the other hand, it is yet stronger than the common assumption

$$
\left|r^{\prime}\right|^{2}+b^{\prime} \leq(1-\delta) c+K_{1} a,
$$

which along with other standard assumptions guarantees that solutions of $D_{t} u=$ $\mathcal{L} u-c u+f$ admit estimates of the first derivatives in $x$ independent of the time interval. 


\section{Discussion of Assumption 2.3}

In a subsequent paper about higher-order derivatives estimates we will impose the following assumption, which trivially implies Assumption 2.3.

Assumption 5.1. We have $m \geq 1$ and there exist a constant $\delta \in(0,1]$ and an operator $\mathcal{K}=\mathcal{K}_{h}(t) \in \mathfrak{K}$, such that

$$
\begin{gathered}
2 m \sum_{\lambda \in \Lambda}\left(\bar{\delta}_{\lambda} \varphi\right) L_{\lambda}^{0} T_{\lambda} \varphi \leq(1-\delta) \sum_{\lambda \in \Lambda} \mathcal{Q}\left(\bar{\delta}_{\lambda} \varphi\right) \\
+K_{1} \mathcal{Q}(\varphi)+2(1-\delta) c \mathcal{K}\left(\sum_{\lambda \in \Lambda}\left|\bar{\delta}_{\lambda} \varphi\right|^{2}\right)
\end{gathered}
$$

on $H_{T}$ for all smooth functions $\varphi$.

In this section we are going to discuss Assumptions 2.3 and 5.1. Here we suppose that only Assumptions 2.1 and 2.2 are satisfied.

Remark 5.1. Condition (5.1) involves a mixture of finite differences and derivatives. It is reasonable to split it into two parts, the combination of which turns out to imply (5.1): For all smooth $\varphi$ we have on $H_{T}$ that

$$
\begin{gathered}
2 m \sum_{\lambda \in \Lambda_{1}}\left(\bar{\delta}_{\lambda} \varphi\right) L_{\lambda}^{0} T_{\lambda} \varphi \leq(1-\delta) \sum_{\lambda \in \Lambda_{1}} \mathcal{Q}\left(\bar{\delta}_{\lambda} \varphi\right) \\
+K_{1} \mathcal{Q}(\varphi)+2(1-\delta) c \mathcal{K}\left(\sum_{\lambda \in \Lambda_{1}}\left|\bar{\delta}_{\lambda} \varphi\right|^{2}\right) ;
\end{gathered}
$$

that is, (5.1) holds with $\tau_{0}=0$ and

$$
\begin{aligned}
& 2 m \sum_{i=1}^{d}\left(D_{i} \varphi\right) L_{i}^{0} \varphi \leq K_{1} \sum_{\lambda \in \Lambda_{1}} \mathcal{Q}\left(\bar{\delta}_{\lambda} \varphi\right) \\
& +(1 / 2) \delta c|D \varphi|^{2}+K_{1} \mathcal{K}\left(\sum_{\lambda \in \Lambda_{1}}\left|\bar{\delta}_{\lambda} \varphi\right|^{2}\right),
\end{aligned}
$$

where and below by $\mathcal{K}$ we denote generic operators (perhaps, depending on $h$ and t) of class $\mathfrak{K}$ and

$$
L_{i}^{0} \varphi=\sum_{\lambda \in \Lambda_{1}}\left(h^{-1} D_{i} q_{\lambda}+D_{i} p_{\lambda}\right) \delta_{\lambda} \varphi .
$$

To show that (5.2) combined with (5.3) implies (5.1) if we choose small $\tau_{0}>0$ appropriately, observe that in terms of $\bar{\delta}_{\ell_{i}}=\tau_{0} D_{i}$, equation (5.3) means that

$$
\begin{aligned}
& 2 m \sum_{\lambda \in \Lambda_{2}}\left(\bar{\delta}_{\lambda} \varphi\right) L_{\lambda}^{0} \varphi \leq K_{1} \tau_{0}^{2} \sum_{\lambda \in \Lambda_{1}} \mathcal{Q}\left(\bar{\delta}_{\lambda} \varphi\right) \\
& \quad+(1 / 2) \delta c \sum_{\lambda \in \Lambda_{2}}\left|\bar{\delta}_{\lambda} \varphi\right|^{2}+K_{1} \tau_{0}^{2} \mathcal{K}\left(\sum_{\lambda \in \Lambda_{1}}\left|\bar{\delta}_{\lambda} \varphi\right|^{2}\right) .
\end{aligned}
$$

By choosing $\tau_{0}$ so that $K_{1} \tau_{0}^{2} \leq \delta / 2$ and $K_{1} \tau_{0}^{2} \leq(1 / 2) \delta c_{0}$, slightly redefining $\mathcal{K}$ to absorb the second term on the right in (5.4), and summing up (5.4) and (5.2) we come to an inequality which is even somewhat stronger than (5.1) if $\delta$ there is replaced with $\delta / 2$, which is irrelevant.

Remark 5.2. It turns out that in a "typical" case out of conditions (5.2) and (5.3) the latter is automatically satisfied. Namely assume that the symmetry condition (S) holds, $q_{\lambda} \geq 0, p_{\lambda} \geq 0$, and $r_{\lambda}:=\sqrt{q_{\lambda}}$ are Lipschitz continuous in $x$ with a 
constant independent of $t$. Then we claim that condition (5.3) is satisfied with any $\delta \in(0,1), \tau_{\lambda} \equiv 1$, and appropriate $K_{1}$ and unit $\mathcal{K}$.

To show this observe that, for any unit $\xi,\left|q_{\lambda(\xi)}\right| \leq N r_{\lambda}$ with $N$ being the doubled Lipschitz constant of $r_{\lambda}$. In particular, by Hölder's inequality,

$$
\left(\sum_{\mu \in \Lambda_{1}} q_{\mu(\xi)} \Delta_{\mu} \varphi\right)^{2} \leq N\left(\sum_{\mu \in \Lambda_{1}} \sqrt{q}_{\mu}\left|\Delta_{\mu} \varphi\right|\right)^{2} \leq N \sum_{\mu \in \Lambda_{1}} \mathcal{Q}\left(\delta_{\mu} \varphi\right)
$$

which allows us to make obvious changes in the estimates of $I_{2}^{(j)}$ in the end of Section 2, one of the changes being that now we can allow $\mathcal{Q}\left(\bar{\delta}_{\lambda} \varphi\right)$ to enter the estimates with as large a constant as we wish.

In the following remark we discuss an estimate which was crucial in the nonlinear setting for establishing a rate of convergence of difference approximations to the true solutions of Bellman's equations in cylindrical domains (see [3]). We will see how using different $\tau_{\lambda}$ can help estimate the derivatives of solutions depending on a parameter.

Remark 5.3. Consider the situation when the coefficients $q_{\lambda}, p_{\lambda}$ and $c$, the free term $f$, and the terminal data $g$ also depend on a parameter $y \in \mathbb{R}$ :

$$
q_{\lambda}=q_{\lambda}(t, z), \quad p_{\lambda}=p_{\lambda}(t, z), \quad c=c(t, z), \quad f=f(t, z), \quad g=g(z),
$$

where $z=(x, y) \in \mathbb{R}^{d+1}$. Assume that these functions and their first derivatives in $z$ are bounded on $H_{T}^{\prime}=[0, T] \times \mathbb{R}^{d+1}$ and continuous in $z$. Assume that $c \geq c_{0}$ for all values of the arguments. Suppose that (15.2) holds on $H_{T}$ for $m=1, \tau_{\lambda} \equiv 1$, any smooth $\varphi(x)$, and any value of the parameter $y$ with $\mathcal{K}$ perhaps depending on $y$ (as well as $h$ and $t$ ). Assume also that the symmetry condition (S) holds, $q_{\lambda} \geq 0$, $p_{\lambda} \geq 0$, and $r_{\lambda}=\sqrt{q_{\lambda}}$ are Lipschitz continuous in $z$ with a constant independent of $t$.

Finally, suppose that in $H_{T}^{\prime}$ we are given a bounded function $u(t, x)=u(t, x, y)$ which satisfies (2.1) in $Q_{T}$ for each value of $y$. Of course, now in (2.1) we write $z$ in place of $x$.

Take an $\varepsilon>0$ and set

$$
T_{\varepsilon}^{y} u(t, x, y)=u(t, x, y+\varepsilon), \quad \delta_{\varepsilon}^{y}=\varepsilon^{-1}\left(T_{\varepsilon}^{y}-1\right) .
$$

We claim that in $[0, T] \times Q \times \mathbb{R}$ it holds that

$$
\left|\delta_{\varepsilon}^{y} u\right| \leq N\left(\sup _{H_{T} \times \mathbb{R}}\left(|f|+\left|D_{z} f\right|\right)+\sup _{\left(\delta^{\prime} Q_{T}\right) \times \mathbb{R}}\left(|u|+\left|\delta_{\varepsilon}^{y} u\right|+\sum_{\lambda \in \Lambda_{1}}\left|\delta_{\lambda} u\right|\right)\right)
$$

if $\varepsilon \in(0, \tau h]$, where $N$ and $\tau \in(0,1]$ depend only on $\delta, c_{0}, K_{1}$, the number of elements in $\Lambda_{1},\left|\Lambda_{1}\right|$, and the Lipschitz constants of $c, r_{\lambda}$, and $p_{\lambda}$ with respect to $z=(x, y)$.

To prove the claim observe that, although equation (2.1) can be considered in $Q_{T}$ as an equation with parameter $y$, we will treat it as an equation in $Q_{T}^{\prime}=Q_{T} \times \mathbb{R}$. Then we denote by $\lambda_{0}$ the positive vector on the $y$-axis having the length $\varepsilon / h$, take a $\tau>0$ to be specified later and introduce

$$
\Lambda_{1}^{\prime}=\Lambda_{1} \cup\left\{\lambda_{0}\right\}, \quad q_{\lambda_{0}}=p_{\lambda_{0}}=0, \quad \tau_{\lambda_{0}}=\tau h / \varepsilon, \quad \tau_{\lambda}=1, \quad \lambda \in \Lambda_{1} .
$$


We now check that Assumption 2.3 is satisfied for the new objects with $m=1$, $\tau_{0}=0$ and $\Lambda_{1}^{\prime}$ in place of $\Lambda$. Owing to the assumption that (5.2) holds, we immediately see that the left-hand side of (2.3) for the new objects is less than

$$
(1-\delta) \sum_{\lambda \in \Lambda_{1}} \mathcal{Q}\left(\delta_{\lambda} \varphi\right)+K_{1} \mathcal{Q}(\varphi)+2(1-\delta) c \mathcal{K}\left(\sum_{\lambda \in \Lambda_{1}}\left|\delta_{\lambda} \varphi\right|^{2}\right)+I,
$$

where $I=I_{1}+I_{2}$,

$$
I_{1}=\left(\bar{\delta}_{\lambda_{0}} \varphi\right) \sum_{\mu \in \Lambda_{1}}\left(\bar{\delta}_{\lambda_{0}} q_{\mu}\right) \Delta_{\mu} T_{\lambda_{0}} \varphi, \quad I_{2}=2\left(\bar{\delta}_{\lambda_{0}} \varphi\right) \sum_{\mu \in \Lambda_{1}}\left(\bar{\delta}_{\lambda_{0}} p_{\mu}\right) \delta_{\mu} T_{\lambda_{0}} \varphi .
$$

Since for smooth $\psi$,

$$
\bar{\delta}_{\lambda_{0}} \psi=\tau \delta_{\varepsilon}^{y} \psi, \quad\left|\bar{\delta}_{\lambda_{0}} \psi\right| \leq \tau \sup |\partial \psi / \partial y|
$$

we have that

$$
\left|I_{2}\right| \leq N \tau \mathcal{K}\left(\sum_{\mu \in \Lambda_{1}^{\prime}}\left|\bar{\delta}_{\mu} \varphi\right|^{2}\right) .
$$

Upon observing the following general properties of finite differences:

$$
h \delta_{\lambda} \delta_{\mu}=\left(T_{\mu}-1\right) \delta_{\lambda}, \quad \Delta_{\mu} T_{\lambda}=-\delta_{\mu} \delta_{-\mu}+\delta_{\lambda} \delta_{\mu}+\delta_{\lambda} \delta_{-\mu}
$$

and combining them with the estimate

$$
\tau^{-1}\left|\bar{\delta}_{\lambda_{0}} q_{\mu}\right|=\left|\delta_{\varepsilon}^{y} q_{\mu}\right|=\left|2 r_{\mu} \delta_{\varepsilon}^{y} r_{\mu}+\varepsilon\left(\delta_{\varepsilon}^{y} r_{\mu}\right)^{2}\right| \leq N\left(\sqrt{q}_{\mu}+h\right)
$$

and (5.5), one easily shows that

$$
\left|I_{1}\right| \leq N \tau\left(\sum_{\lambda \in \Lambda_{1}^{\prime}} \mathcal{Q}\left(\delta_{\lambda} \varphi\right)+\mathcal{K}\left(\sum_{\mu \in \Lambda_{1}^{\prime}}\left|\bar{\delta}_{\mu} \varphi\right|^{2}\right)\right) .
$$

Since $\varepsilon \leq \tau h$, we have that $\left|\delta_{\lambda} \varphi\right| \leq\left|\bar{\delta}_{\lambda} \varphi\right|$ on $\Lambda_{1}^{\prime}$ and the above estimates show how to choose $\tau \in(0,1]$ in order for Assumption 2.3 to be satisfied indeed.

By applying Theorem 2.1 (and Remark 2.4) we finish proving our claim. The only point which is perhaps worth noting is that by Theorem 2.1 the constant $N$ from (5.6) also depends on $\left|\Lambda_{1}^{\prime}\right|$. However, $\left|\Lambda_{1}^{\prime}\right|^{2}=\left|\Lambda_{1}\right|^{2}+\tau^{2}$.

\section{Discussion of Assumptions 2.3 And 5.1 In Case that $\tau_{\lambda}=1, \lambda \in \Lambda_{1}$}

Here we suppose that only Assumptions 2.1 and 2.2 are satisfied. Everywhere below we set $\tau_{\lambda}=1$ for all $\lambda \in \Lambda_{1}$.

Remark 6.1. In some situations (see, for instance, Remark 6.2) it is desirable to have $p_{\lambda}$ large. It turns out that one can modify $p_{\lambda}$ to achieve this if $L_{h}$ are used to approximate a differential operator. More precisely, suppose that the symmetry assumption $(\mathrm{S})$ is satisfied. Then the operators $L_{h}$ can be regarded as finitedifference approximations of $\mathcal{L}$ (see (2.8)) in the sense that for any smooth $\varphi$ we have $L_{h} \varphi \rightarrow \mathcal{L} \varphi$ as $h \downarrow 0$. If we are only interested in this property, then we can always assume that $p_{\lambda} \geq K_{1}$.

Indeed, if we do not have this inequality, then we take a sufficiently large constant $K_{2}$ (independent of $h$ ) and redefine $p_{\lambda}$ as $p_{\lambda}+K_{2}$. This will not violate the convergence $L_{h} \varphi \rightarrow \mathcal{L} \varphi$ since

$$
2 \sum_{\lambda \in \Lambda_{1}} \delta_{\lambda} \varphi=\sum_{\lambda \in \Lambda_{1}}\left[\delta_{\lambda}+\delta_{-\lambda}\right] \varphi \rightarrow 0
$$

if $\varphi$ is smooth. 
The following lemma is often used below and in the continuation of the present paper.

Lemma 6.1. Let $\alpha_{\lambda \mu}$ be a nonnegative function on $\mathbb{R}^{d}$ for each $\mu \in \Lambda_{1}$ and $\lambda$ from a finite set of indices $\Lambda^{\prime}$. Assume that

$$
\sum_{\mu \in \Lambda_{1}} \sup _{\lambda \in \Lambda^{\prime}} \alpha_{\lambda \mu} \leq C
$$

on $\mathbb{R}^{d}$ for some function $C$. Then there is a $\mathcal{K} \in \mathfrak{K}$ such that

$$
h^{2} \sum_{\mu \in \Lambda_{1}, \lambda \in \Lambda^{\prime}} \alpha_{\lambda \mu}\left(\delta_{\mu} f_{\lambda}\right)^{2} \leq 4 C \mathcal{K}\left(\sum_{\lambda \in \Lambda^{\prime}} f_{\lambda}^{2}\right)
$$

on $\mathbb{R}^{d}$ for any bounded Borel function $f=f_{\lambda}$ given on $\mathbb{R}^{d}$ for $\lambda \in \Lambda^{\prime}$.

Proof. Using

$$
h^{2}\left(\delta_{\mu} f_{\lambda}\right)^{2}=\left(\left(T_{\mu}-1\right) f_{\lambda}\right)^{2} \leq 2\left(T_{\mu} f_{\lambda}\right)^{2}+2 f_{\lambda}^{2}=2\left(T_{\mu}+1\right) f_{\lambda}^{2},
$$

we can estimate from above the left-hand side of (6.1) by

$$
2 \sum_{\mu \in \Lambda_{1}} C_{\mu}\left(T_{\mu}+1\right) \sum_{\lambda \in \Lambda^{\prime}} f_{\lambda}^{2},
$$

where $C_{\mu}:=\sup _{\lambda \in \Lambda^{\prime}} \alpha_{\lambda \mu}$. Hence we get 6.1) with $\mathcal{K} \in \mathfrak{K}$ defined by

$$
\mathcal{K}(f)=\frac{1}{2 C} \sum_{\mu \in \Lambda_{1}} C_{\mu}\left(T_{\mu}+1\right) f .
$$

The lemma is proved.

Remark 6.2. One can give sufficient conditions for (5.2) to hold without involving test functions $\varphi$, which makes them more "explicit" and in combination with Remark 5.2 covers many situations when Assumption 5.1] is relatively easy to check. One set of these "explicit" conditions is given in this remark. Here we also show why the operators of class $\mathfrak{K}$ are useful and how the presence of $h p_{\lambda}$ in $\chi_{\lambda}$ entering the operator $\mathcal{Q}$ on the right of (5.2) may help.

Suppose that $\Lambda_{1}=-\Lambda_{1}$ and $q_{\lambda}=q_{-\lambda} \geq 0$ and set $r_{\lambda}=\sqrt{q_{\lambda}}$. Take a $\delta \in(0,1 / 4)$ and assume that on $H_{T}$ there are functions $r_{\lambda \mu}, p_{\lambda \mu} \geq 0, \lambda, \mu \in \Lambda_{1}$, such that

$$
\begin{array}{ll}
h^{2}\left(\delta_{\lambda} r_{\mu}\right)^{2} \leq \delta\left(\chi_{\mu}+\chi_{\lambda}\right)+h^{2} r_{\lambda \mu}^{2}, & \sum_{\mu \in \Lambda_{1}} \sup _{\lambda \in \Lambda_{1}} r_{\lambda \mu}^{2} \leq 2 \delta c, \\
h^{2}\left|\delta_{\lambda} p_{\mu}\right| \leq \delta^{2}\left(\chi_{\mu}+\chi_{\lambda}\right)+\delta h^{2} p_{\lambda \mu}, & \sum_{\mu \in \Lambda_{1}} \sup _{\lambda \in \Lambda_{1}} p_{\lambda \mu} \leq \delta c .
\end{array}
$$

By virtue of Remark 6.1 if $L_{h}$ are used for approximating $\mathcal{L}$, we can change these operators and have (6.2) and (6.3) satisfied with $r_{\lambda \mu}=p_{\lambda \mu}=0$ for sufficiently small $h$, provided that the Lipschitz constants in $x$ of $r_{\lambda}$ and $p_{\lambda}$ are bounded with respect to $t$. 
For a function $\xi_{\lambda}$ given on $\Lambda_{1}$ let us write

$$
|\xi|^{2}=\sum_{\lambda \in \Lambda_{1}}\left|\xi_{\lambda}\right|^{2}
$$

Then it turns out that condition (5.2) is satisfied if on $H_{T}$ for all functions $\xi_{\lambda}$ we have

$$
\begin{aligned}
& 10 m^{2}(1-4 \delta)^{-1} J_{1}+2 m^{2}(1-4 \delta)^{-1} J_{2} \\
& +2 \delta m^{2} \sum_{\lambda, \mu \in \Lambda_{1}} \xi_{\lambda}^{2}\left|\delta_{\lambda} p_{\mu}\right|+2 m \sum_{\lambda, \mu \in \Lambda_{1}} \xi_{\lambda} \xi_{\mu}\left(\delta_{\lambda} p_{\mu}+\left(\delta_{\lambda} r_{\mu}\right)^{2}\right) \\
& \leq(2-8 \delta) c|\xi|^{2}+K_{1} \sum_{\lambda \in \Lambda_{1}} \xi_{\lambda}^{2} \chi_{\lambda}+\delta h^{-2} \sum_{\lambda \in \Lambda_{1}} \chi_{\lambda}\left|\xi_{\lambda}+\xi_{-\lambda}\right|^{2},
\end{aligned}
$$

where

$$
J_{1}=\sum_{\mu, \lambda \in \Lambda_{1}} \xi_{\lambda}^{2}\left(\delta_{\lambda} r_{\mu}\right)^{2}, \quad J_{2}=\sum_{\mu \in \Lambda_{1}}\left(\sum_{\lambda \in \Lambda_{1}} \xi_{\lambda} \delta_{\lambda} r_{\mu}\right)^{2} .
$$

To prove this, use formulas (5.7). Also drop the summation sign having repeated indices in $\Lambda_{1}$ to see that

$$
\begin{aligned}
& 2\left(\delta_{\lambda} \varphi\right) Q_{\lambda} T_{\lambda} \varphi=\left(\delta_{\lambda} \varphi\right)\left(\delta_{\lambda} q_{\mu}\right)\left(\delta_{\lambda} \delta_{\mu}+\delta_{\lambda} \delta_{-\mu}+\Delta_{\mu}\right) \varphi \\
& \quad=2 \xi_{\lambda}\left(\delta_{\lambda} q_{\mu}\right) \delta_{\lambda} \delta_{\mu} \varphi-\xi_{\lambda}\left(\delta_{\lambda} q_{\mu}\right) \delta_{\mu} \delta_{-\mu} \varphi=I_{11}+I_{12}+I_{21}+I_{22},
\end{aligned}
$$

where $\xi_{\lambda}=\delta_{\lambda} \varphi$ and

$$
\begin{aligned}
& I_{11}=4 \xi_{\lambda}\left(\delta_{\lambda} r_{\mu}\right) r_{\mu} \delta_{\lambda} \delta_{\mu} \varphi, \quad I_{12}=2 h \xi_{\lambda}\left(\delta_{\lambda} r_{\mu}\right)^{2} \delta_{\lambda} \delta_{\mu} \varphi \\
& I_{21}=-2 \xi_{\lambda}\left(\delta_{\lambda} r_{\mu}\right) r_{\mu} \delta_{-\mu} \delta_{\mu} \varphi, \quad I_{22}=h \xi_{\lambda}\left(\delta_{\lambda} r_{\mu}\right)^{2} \Delta_{\mu} \varphi=2 \xi_{\lambda}\left(\delta_{\lambda} r_{\mu}\right)^{2} \xi_{\mu} .
\end{aligned}
$$

Before starting to estimate $I_{i j}$, we note that as $h \downarrow 0$ the terms $I_{11}, I_{22}$, and $I_{12}$ disappear if $\varphi$ is twice continuously differentiable due to the symmetry of $\Lambda_{1}$. In that case there is no need to estimate them. For fixed $h$ they are present and estimating them is only possible under stronger assumptions than in the case of partial differential equations.

Now notice that by Young's inequality,

$$
m I_{11} \leq(1 / 2)(1-4 \delta) I+8 m^{2}(1-4 \delta)^{-1} J_{1},
$$

where

$$
I=\sum_{\lambda \in \Lambda_{1}} \mathcal{Q}\left(\delta_{\lambda} \varphi\right)=\sum_{\lambda, \mu \in \Lambda_{1}} \chi_{\mu}\left|\delta_{\mu} \delta_{\lambda} \varphi\right|^{2} .
$$

Similarly,

$$
m I_{21} \leq(1 / 2)(1-4 \delta) I+2 m^{2}(1-4 \delta)^{-1} J_{2} .
$$

Next, owing to (6.2),

$$
\begin{aligned}
& m I_{12}=2 m\left(\xi_{\lambda} \delta_{\lambda} r_{\mu}\right)\left(h\left(\delta_{\lambda} r_{\mu}\right) \delta_{\lambda} \delta_{\mu} \varphi\right) \\
& \leq 2 m^{2} J_{1}+(1 / 2) h^{2} \sum_{\lambda, \mu \in \Lambda_{1}}\left(\delta_{\lambda} r_{\mu}\right)^{2}\left(\delta_{\lambda} \delta_{\mu} \varphi\right)^{2} \\
& \leq 2 m^{2} J_{1}+\delta I+(1 / 2) h^{2} \sum_{\lambda, \mu \in \Lambda_{1}} r_{\lambda \mu}^{2}\left(\delta_{\lambda} \delta_{\mu} \varphi\right)^{2}
\end{aligned}
$$

where the last term by virtue of Lemma 6.1 is estimated by

$$
4 \delta c \mathcal{K}\left(\sum_{\lambda \in \Lambda_{1}}\left(\delta_{\lambda} \varphi\right)^{2}\right)
$$


By collecting the above estimates we obtain

$$
\begin{aligned}
& 2 m\left(\delta_{\lambda} \varphi\right) Q_{\lambda} T_{\lambda} \varphi \leq(1-3 \delta) I+10 m^{2}(1-4 \delta)^{-1} J_{1} \\
& \quad+2 m^{2}(1-4 \delta)^{-1} J_{2}+2 m\left(\delta_{\lambda} r_{\mu}\right)^{2} \xi_{\lambda} \xi_{\mu}+4 \delta c \mathcal{K}\left(\sum_{\lambda \in \Lambda_{1}}\left(\delta_{\lambda} \varphi\right)^{2}\right) .
\end{aligned}
$$

Next,

$$
2\left(\delta_{\lambda} \varphi\right) P_{\lambda} T_{\lambda} \varphi=2 \xi_{\lambda}\left(\delta_{\lambda} p_{\mu}\right) \xi_{\mu}+2 h \xi_{\lambda}\left(\delta_{\lambda} p_{\mu}\right) \delta_{\lambda} \delta_{\mu} \varphi
$$

where the last term is majorized by

$$
2 \delta m \sum_{\lambda, \mu \in \Lambda_{1}} \xi_{\lambda}^{2}\left|\delta_{\lambda} p_{\mu}\right|+(1 / 2) m^{-1} \delta^{-1} h^{2} \sum_{\lambda, \mu \in \Lambda_{1}}\left|\delta_{\lambda} p_{\mu}\right|\left(\delta_{\lambda} \delta_{\mu} \varphi\right)^{2} .
$$

We use assumption (6.3) and proceed as while estimating $I_{12}$. Then we see that

$$
\begin{aligned}
& 2 m\left(\delta_{\lambda} \varphi\right) P_{\lambda} T_{\lambda} \varphi \leq 2 m \xi_{\lambda}\left(\delta_{\lambda} p_{\mu}\right) \xi_{\mu} \\
& \quad+2 \delta m^{2} \sum_{\lambda, \mu \in \Lambda_{1}} \xi_{\lambda}^{2}\left|\delta_{\lambda} p_{\mu}\right|+\delta I+2 \delta c \mathcal{K}\left(\sum_{\lambda \in \Lambda_{1}}\left(\delta_{\lambda} \varphi\right)^{2}\right) .
\end{aligned}
$$

Finally, upon combining (6.6) with (6.5) we obtain

$$
\begin{aligned}
& 2 m\left(\delta_{\lambda} \varphi\right) L_{\lambda}^{0} T_{\lambda} \varphi \leq(1-2 \delta) I+10 m^{2}(1-4 \delta)^{-1} J_{1}+2 m^{2}(1-4 \delta)^{-1} J_{2} \\
& \quad+2 \delta m^{2} \sum_{\lambda, \mu \in \Lambda_{1}} \xi_{\lambda}^{2}\left|\delta_{\lambda} p_{\mu}\right|+6 \delta c \mathcal{K}\left(\sum_{\lambda \in \Lambda_{1}}\left(\delta_{\lambda} \varphi\right)^{2}\right) \\
& \quad+2 m \xi_{\lambda}\left(\left(\delta_{\lambda} r_{\mu}\right)^{2}+\left(\delta_{\lambda} p_{\mu}\right)\right) \xi_{\mu} .
\end{aligned}
$$

We use the fact that $h^{-1}\left(\delta_{\lambda}+\delta_{-\lambda}\right)=-\delta_{\lambda} \delta_{-\lambda}$, use assumption (6.4), and take into account that for any $\mathcal{K}^{\prime} \in \mathfrak{K}$,

$$
(2-8 \delta) c \sum_{\lambda \in \Lambda_{1}}\left|\delta_{\lambda} \varphi\right|^{2}+6 \delta c \mathcal{K}^{\prime}\left(\sum_{\lambda \in \Lambda_{1}}\left|\delta_{\lambda} \varphi\right|^{2}\right)=(2-2 \delta) c \mathcal{K}\left(\sum_{\lambda \in \Lambda_{1}}\left|\delta_{\lambda} \varphi\right|^{2}\right)
$$

with an appropriate $\mathcal{K} \in \mathfrak{K}$. Then we estimate the right-hand side of (6.7) by

$$
(1-\delta) I+K_{1} \mathcal{Q}(\varphi)+(2-2 \delta) c \mathcal{K}\left(\sum_{\lambda \in \Lambda_{1}}\left(\delta_{\lambda} \varphi\right)^{2}\right),
$$

and we see that (5.2) is satisfied indeed.

The following remark will be used in a subsequent paper pertaining to estimating higher-order derivatives. There we will use a modification of (6.4).

Remark 6.3. It is easy to see that if (6.2) and (6.3) hold and we assume that inequality (6.4) is satisfied with an additional term $\Psi(t, x, \xi)$ on its left-hand side for some function $\Psi$ of $t, x$ and $\xi=\left(\xi_{\lambda}\right)_{\lambda \in \Lambda_{1}}$, then inequality (5.2) holds with the additional term $\Psi\left(t, x,\left(\delta_{\lambda} \varphi\right)_{\lambda \in \Lambda_{1}}\right)$ on its left-hand side.

Remark 6.4. Here we show that if $c_{0}$ is sufficiently large and $q_{\lambda}$ are smooth, then typically Assumption 2.3 is satisfied. Assume (S), assume that $q_{\lambda} \geq 0, p_{\lambda} \geq 0$ and let $m \geq 2$. Then it turns out that Assumption 2.3 is satisfied for $\delta=1 / 10$ and appropriate $\tau_{0}>0$ or $\tau_{0}=0$ if $c_{0}$ is sufficiently large (independently of $h$ ).

Indeed, it is well known that the Lipschitz constant of the square root of a nonnegative twice continuously differentiable function $w(x)$ is controlled by the supremums of its second-order derivatives. Therefore, Remark 6.2 (where we take $r_{\lambda \mu}=\delta_{\lambda} r_{\mu}$ and $\left.p_{\lambda \mu}=10\left|\delta_{\lambda} p_{\mu}\right|\right)$ immediately implies that condition (5.2) is satisfied if $c_{0}$ is large enough. 
That condition (5.3) is satisfied follows from Remark 5.2 and again from the fact that for twice continuously differentiable $w \geq 0$ on $\mathbb{R}^{d}$ we have

$$
|D w|^{2} \leq 2 w \sup _{x \in \mathbb{R}^{d},|\xi|=1}\left|w_{(\xi)(\xi)}(x)\right| .
$$

At this point we do not even need large $c_{0}$. Referring to Remark 5.1 we obtain what we have claimed.

Actually, above in this remark we used that $m \geq 2$ only to guarantee that $r_{\lambda}$ are Lipschitz continuous in $x$ with a constant $N^{\prime}$ independent of $t$. If we just assumed this last property, then our argument concerning (5.2) would become even shorter. In addition, what was said about (5.3) is still valid.

Remark 6.5. Under the symmetry assumption (S) and the assumption that $q_{\lambda} \geq 0$ and $p_{\lambda} \geq 0$ one can give a rougher condition without using $\xi_{\lambda}$ and implying (6.4) with $m=1$ and sufficiently small $\delta$. Then (5.2) will be satisfied as long as conditions (6.2) and (6.3) are.

By the way, recall that, for all small $h$, one can always satisfy conditions (6.2) and (6.3) on the account of modifying if necessary $p_{\lambda}$ if the Lipschitz constants of $r_{\lambda}$ in $x$ are bounded in $t$ (see Remark 6.2).

By the inequality

$$
\sum_{\lambda, \mu \in \Lambda_{1}} \eta_{\lambda} \eta_{\mu} \alpha_{\lambda \mu} \leq \frac{1}{2} \sum_{\lambda \in \Lambda_{1}} \eta_{\lambda}^{2} \sum_{\mu \in \Lambda_{1}}\left|\alpha_{\lambda \mu}+\alpha_{\mu \lambda}\right|
$$

we have

$$
\begin{aligned}
& \sum_{\lambda, \mu \in \Lambda_{1}} \xi_{\lambda} \xi_{\mu}\left(\delta_{\lambda} p_{\mu}+\left(\delta_{\lambda} r_{\mu}\right)^{2}\right) \\
& \quad \leq \frac{1}{2} \sum_{\lambda \in \Lambda_{1}} \xi_{\lambda}^{2} \sum_{\mu \in \Lambda_{1}}\left|\delta_{\lambda} p_{\mu}+\delta_{\mu} p_{\lambda}+\left(\delta_{\lambda} r_{\mu}\right)^{2}+\left(\delta_{\mu} r_{\lambda}\right)^{2}\right|, \\
& J_{2}=\sum_{\lambda, \nu, \in \Lambda_{1}} \xi_{\lambda} \xi_{\nu} \sum_{\mu \in \Lambda_{1}}\left(\delta_{\lambda} r_{\mu}\right)\left(\delta_{\nu} r_{\mu}\right) \leq \sum_{\lambda \in \Lambda_{1}} \xi_{\lambda}^{2} \sum_{\nu \in \Lambda_{1}}\left|\sum_{\mu \in \Lambda_{1}}\left(\delta_{\lambda} r_{\mu}\right) \delta_{\nu} r_{\mu}\right| .
\end{aligned}
$$

A simple argument based on the above estimates and continuity shows that (6.4) holds with $m=1$ and a small $\delta>0$ if for any $\lambda \in \Lambda_{1}$,

$$
\begin{aligned}
10 & \sum_{\mu \in \Lambda_{1}}\left(\delta_{\lambda} r_{\mu}\right)^{2}+2 \sum_{\nu \in \Lambda_{1}}\left|\sum_{\mu \in \Lambda_{1}}\left(\delta_{\lambda} r_{\mu}\right) \delta_{\nu} r_{\mu}\right| \\
& +\sum_{\mu \in \Lambda_{1}}\left|\delta_{\lambda} p_{\mu}+\delta_{\mu} p_{\lambda}+\left(\delta_{\lambda} r_{\mu}\right)^{2}+\left(\delta_{\mu} r_{\lambda}\right)^{2}\right| \leq c+K_{1} q_{\lambda} .
\end{aligned}
$$

Condition (6.8) basically means that if for a $\lambda \in \Lambda_{1}$ at some point in $H_{T}$ the value $q_{\lambda}$ is small, then either $\delta_{\lambda} r_{\mu}, \delta_{\mu} r_{\lambda}, \delta_{\mu} p_{\lambda}$, and $\delta_{\lambda} p_{\mu}$ should be small or $c$ should be large at this point. As the point varies, the dominating terms may change roles.

Remark 6.6. There are cases when it is preferable to keep the last term on the left in (6.4) as is using the fact that sometimes it can be large negative.

To see a reason for that, let $m=d=1$, take a constant $\theta \geq 1$ and define $\Lambda_{1}=\{ \pm 1\}$

$$
q_{\lambda} \equiv 0, \quad p_{1}(t, x)=(1 / 2) b(x)+\theta, \quad p_{-1}=-(1 / 2) b(x)+\theta,
$$

where $b(x)$ is a decreasing function with bounded derivative such that $|b| \leq 1$. Observe that for any $\theta$ we have $L_{h}^{0} \varphi \rightarrow b \varphi^{\prime}$ as $h \downarrow 0$ if $\varphi$ is smooth. 
Now notice that condition (6.2) is trivially satisfied since $r_{\lambda} \equiv 0$. Condition (6.3) is satisfied with any $\delta>0$ and $p_{\lambda \mu}=0$ if $h \leq 1$ and $\theta \geq 100 \sup \left|b^{\prime}\right|+1$ since

$$
\chi_{\lambda}=h p_{\lambda} \geq h(\theta-|b|) \geq h(\theta-1) .
$$

The left-hand side of (6.4) is one half of

$$
4 \delta \sum_{i= \pm 1} \xi_{i}^{2}\left|\delta_{i} b\right|+\left(\xi_{1}-\xi_{-1}\right)^{2}\left[\delta_{1} b-\delta_{-1} b\right]+\left(\xi_{1}^{2}-\xi_{-1}^{2}\right)\left(\delta_{1} b+\delta_{-1} b\right)
$$

Here the middle term is nonpositive since $b$ is decreasing. Also the last term is majorized by

$$
N\left|\xi_{-1}+\xi_{1}\right|\left|\xi_{1}-\xi_{-1}\right| \leq N h\left(\xi_{1}^{2}+\xi_{-1}^{2}\right)+N h^{-1}\left(\xi_{-1}+\xi_{1}\right)^{2} .
$$

Furthermore, concerning the right-hand side of (6.4) observe that

$$
\sum_{\lambda} \xi_{\lambda}^{2} \chi_{\lambda} \geq h(\theta-1) \sum_{\lambda} \xi_{\lambda}^{2}, \quad h^{-2} \sum_{\lambda} \chi_{\lambda}\left(\xi_{\lambda}+\xi_{-\lambda}\right)^{2} \geq h^{-1}(\theta-1)\left(\xi_{1}+\xi_{-1}\right)^{2} .
$$

It follows easily that, no matter how small $c_{0}$ is, for sufficiently small $\delta$ and large $\theta$, condition (6.4) and, by Remark 6.2. condition (5.2) are satisfied. This along with the almost obvious fact that (5.3) holds shows that Assumption 2.3 is satisfied as well.

To finish the remark notice that if we tried to check condition (6.8) we would fail to do that for small $h$ no matter how large $\theta$ is unless $c$ is large enough.

Remark 6.7. We continue the analysis of the one-dimensional situation started in Remark 6.6. So, we assume that $d=m=1$ and we have in mind approximating an operator $\mathcal{L} \varphi(x)=(1 / 2) a(x) \varphi^{\prime \prime}(x)+b(x) \varphi^{\prime}(x)$. As in Remark 6.6 we assume that $|b| \leq 1$ and, in addition, assume that $a \geq 0$ and $r:=\sqrt{a}$ and $b$ are one time differentiable with derivatives uniformly continuous on $\mathbb{R}$. We show that (6.4) follows from 6.11 below.

As in Remark 6.6 take $\Lambda_{1}=\{ \pm 1\}$ and define $p_{ \pm 1}$ for an appropriate $\theta$. Then also set $r_{\mu}=r$. Now observe that both parts of (6.4) are order-two homogeneous functions of $\xi_{\lambda}$. Therefore, it suffices to check (6.4) assuming that

$$
\sum_{\lambda \in \Lambda_{1}} \xi_{\lambda}^{2}=1
$$

Then, due to the assumption that $r^{\prime}$ and $b^{\prime}$ are uniformly continuous on $\mathbb{R}$, it is not hard to see that

$$
\begin{gathered}
J_{1} \sim 2\left(r^{\prime}\right)^{2}, \quad J_{2} \sim 2\left(r^{\prime}\right)^{2}\left(\xi_{1}-\xi_{-1}\right)^{2} \\
\sum_{\lambda, \mu \in \Lambda_{1}} \xi_{\lambda} \xi_{\mu}\left(\delta_{\lambda} r_{\mu}\right)^{2} \sim\left(r^{\prime}\right)^{2}\left(\xi_{1}+\xi_{-1}\right)^{2}
\end{gathered}
$$

where by $\alpha \sim \beta$ we mean that for small $h$ the difference $|\alpha-\beta|$ can be absorbed into $c \geq c_{0}>0$ with as small a coefficient as we wish. Therefore, upon recalling the estimates from Remark 6.6 we see that condition (6.4) is satisfied for all small $h$ if

$$
\begin{aligned}
& 20(1-4 \delta)^{-1}\left(r^{\prime}\right)^{2}+4(1-4 \delta)^{-1}\left(r^{\prime}\right)^{2}\left(\xi_{1}-\xi_{-1}\right)^{2}+2\left(r^{\prime}\right)^{2}\left(\xi_{1}+\xi_{-1}\right)^{2} \\
& \quad+2 \delta\left|b^{\prime}\right|+\left(\xi_{1}-\xi_{-1}\right)^{2} b^{\prime} \leq(2-9 \delta) c+K_{1} a+\delta h^{-1}(\theta-1)\left(\xi_{1}+\xi_{-1}\right)^{2} .
\end{aligned}
$$

On account of (assumption (6.9) and) the presence of $h^{-1}(\theta-1)\left(\xi_{1}+\xi_{-1}\right)^{2}$ on the right of (6.10), it suffices to check (6.10) for small $h$ assuming that the inequality 
$\left|\xi_{1}+\xi_{-1}\right| \leq h^{1 / 2}$ holds. It follows that (6.10) holds for small $h$ if it holds with $(2-10 \delta) c$ in place of $(2-9 \delta) c$ but only for $\xi_{\lambda}$ satisfying $\xi_{1}=-\xi_{-1}$. In that case $\left(\xi_{1}-\xi_{-1}\right)^{2}=4 \xi_{1}^{2}=2$ and (6.10) holds if

$$
28(1-4 \delta)^{-1}\left(r^{\prime}\right)^{2}+2 \delta\left|b^{\prime}\right|+2 b^{\prime} \leq(2-10 \delta) c+K_{1} a .
$$

Since we would be satisfied if (6.4) held with at least one $\delta>0$, we see that, under the assumption of the present remark, (6.4) (perhaps with different $\delta$ and $K_{1}$ ) is indeed satisfied for small $h$ if

$$
14\left(r^{\prime}\right)^{2}+b^{\prime} \leq(1-\delta) c+K_{1} a .
$$

Remark 6.8. There are multi-dimensional analogs of the situation in Remark 6.6. For instance, let $U(x)$ be a concave function with bounded derivatives and assume that

$$
p_{\mu}=(D U, \mu)+\theta
$$

where $\theta \geq \max _{\mu \in \Lambda_{1}}|\mu| \sup |D U|$, so that $p_{\mu} \geq 0$. Then for small $h$ we have

$$
\sum_{\lambda, \mu \in \Lambda_{1}} \xi_{\lambda} \xi_{\mu} \delta_{\lambda} p_{\mu} \sim \sum_{\lambda, \mu \in \Lambda_{1}} \xi_{\lambda} \xi_{\mu}\left(D^{2} U \mu, \lambda\right)=\left(D^{2} U \eta, \eta\right) \leq 0,
$$

where $\eta=\sum \xi_{\lambda} \lambda$.

Remark 6.9. In Remark 5.2 we analyzed when (5.3) is satisfied assuming the symmetry condition. Here we will see that some kind of symmetry is also necessary for (5.3) to hold.

Recall that $\delta_{\lambda}=\delta_{h, \lambda}$ and assume that (5.3) holds for all small $h>0$ with $\mathcal{K}$ perhaps depending on $h$. Then it turns out that for all $t \in[0, \infty)$,

$$
\sum_{\lambda \in \Lambda_{1}} \lambda q_{\lambda}(t, x) \text { is independent of } x .
$$

To show this observe that, since the values of the first derivatives of $\varphi$ at a fixed point have nothing to do with the increments of $\varphi$, (5.3) is equivalent to saying that

$$
\sum_{i=1}^{d}\left(L_{i}^{0} \varphi\right)^{2} \leq N\left(\sum_{\lambda, \nu \in \Lambda_{1}} \chi_{\lambda}\left|\delta_{\lambda} \delta_{\nu} \varphi\right|^{2}+\mathcal{K}\left(\sum_{\lambda \in \Lambda_{1}}\left|\delta_{\lambda} \varphi\right|^{2}\right)\right),
$$

where the constant $N$ can be easily computed given $\sup c$ and $K_{1}$. It follows that

$$
\sum_{i=1}^{d}\left(L_{i}^{0} \varphi\right)^{2} \leq N\left(\sum_{\lambda, \nu \in \Lambda_{1}} \chi_{\lambda}\left|\delta_{\lambda} \delta_{\nu} \varphi\right|^{2}+\sup _{\mathbb{R}^{d}} \sum_{\lambda \in \Lambda_{1}}\left|\delta_{\lambda} \varphi\right|^{2}\right) .
$$

Now multiply (6.13) by $h^{2}$, let $h \downarrow 0$, and observe that $\delta_{\lambda} \delta_{\nu} \varphi$ and $\delta_{\lambda} \varphi$ are bounded since $\varphi$ is smooth. Then we obtain

$$
\sum_{i=1}^{d}\left[\left(D_{j} \varphi(x)\right) \sum_{\mu \in \Lambda_{1}} D_{i} q_{\mu}(t, x) \mu^{j}\right]^{2}=0,
$$

which leads to the conclusion that for smooth $\varphi$ and $i=1, \ldots, d$,

$$
\left(D_{j} \varphi(x)\right) \sum_{\mu \in \Lambda_{1}} D_{i} q_{\mu}(t, x) \mu^{j}=0 .
$$

This is equivalent to saying that (6.12) holds. 
Remark 6.10. Here we show that assumption (5.3) imposes some nontrivial conditions on the structure of $\Lambda_{1}$ and $q_{\lambda}$. Additionally to Assumptions 2.1 and 2.2 suppose that

$$
\lambda+\nu \notin \Lambda_{1} \quad \forall \lambda, \nu \in \Lambda_{1} .
$$

It turns out that in this case (5.3) is satisfied for all small $h$ only if for any $\lambda \in \Lambda_{1}$,

(i) either $-\lambda \in \Lambda_{1}$ and $q_{\lambda}(t, x)=q_{-\lambda}(t, x)+r_{\lambda}(t)$ for a function $r_{\lambda}(t)$ independent of $x$,

(ii) or $-\lambda \notin \Lambda_{1}$ and $q_{\lambda}$ is independent of $x$.

In particular,

$$
Q_{\nu} \varphi=(1 / 2) \sum_{\lambda \in \Lambda_{1} \cap\left(-\Lambda_{1}\right)}\left(\delta_{\nu} q_{\lambda}\right) \Delta_{\lambda} \varphi \quad\left(\sum_{\emptyset} \ldots:=0\right) .
$$

We may concentrate on proving our claim assuming that $q_{\lambda}$ is independent of t. As we have pointed out in Remark 6.9, condition (5.3) implies (6.13). We write the latter at $x=0$, substitute $\varphi(x / h)$ in place of $\varphi$ and let $h \downarrow 0$. Then by just comparing the powers of $h$ in different terms we obtain that for a constant $N$ and for any $i=1, \ldots, d$ and $\varphi$ we have

$$
\sum_{\lambda \in \Lambda_{1}}\left(D_{i} q_{\lambda}(0)\right) \varphi(\lambda) \leq N\left(\sum_{\lambda, \nu \in \Lambda_{1}}\left|\delta_{1, \lambda} \delta_{1, \nu} \varphi(0)\right|^{2}\right)^{1 / 2} .
$$

We see that the linear function (of $\varphi$ ) on the left provides a supporting plane at the origin for the convex function on the right. Consequently, there are some constants $q_{\lambda, \nu}$ such that for all $\varphi$,

$$
\begin{aligned}
& \sum_{\lambda \in \Lambda_{1}}\left(D_{i} q_{\lambda}(0)\right) \varphi(\lambda)=\sum_{\lambda, \nu \in \Lambda_{1}} q_{\lambda, \nu} \delta_{1, \lambda} \delta_{1, \nu} \varphi(0) \\
& \quad=\sum_{\lambda, \nu \in \Lambda_{1}} q_{\lambda, \nu}[\varphi(\lambda+\nu)+\varphi(0)-\varphi(\lambda)-\varphi(\nu)] .
\end{aligned}
$$

Without losing generality we may assume that

$$
q_{\lambda, \nu}=q_{\nu, \lambda}
$$

and split the sum on the right in (6.16) into two parts: the first part with the summation over $\lambda, \nu$ such that $\lambda+\nu=0$ and the second part for $\lambda+\nu \neq 0$. According to assumption (6.14) the terms $\varphi(\lambda+\nu)$ in the second part do not appear elsewhere in (6.16). It follows that $q_{\lambda, \nu}=0$ if $\lambda+\nu \neq 0$, so that

$$
\sum_{\lambda \in \Lambda_{1}}\left(D_{i} q_{\lambda}(0)\right) \varphi(\lambda)=\sum_{\lambda \in \Lambda_{1}} q_{\lambda,-\lambda}[2 \varphi(0)-\varphi(\lambda)-\varphi(-\lambda)]
$$

Here the expression on the right is symmetric with respect to the transformation $\varphi(x) \rightarrow \varphi(-x)$. Thus,

$$
\sum_{\lambda \in \Lambda_{1}}\left(D_{i} q_{\lambda}(0)\right) \varphi(\lambda)=\sum_{\lambda \in \Lambda_{1}}\left(D_{i} q_{\lambda}(0)\right) \varphi(-\lambda) .
$$

We obtained this relation at the origin. Similarly, for any $x$,

$$
\sum_{\lambda \in \Lambda_{1}}\left(D_{i} q_{\lambda}(x)\right) \varphi(\lambda)=\sum_{\lambda \in \Lambda_{1}}\left(D_{i} q_{\lambda}(x)\right) \varphi(-\lambda) .
$$


Fix a $\lambda_{0} \in \Lambda_{1}$ and take a $\varphi$ which is 1 at $\lambda=\lambda_{0}$ and zero otherwise. Then (6.17) shows that

(i) either $-\lambda_{0} \in \Lambda_{1}$ and then $D q_{\lambda_{0}}(x)=D q_{-\lambda_{0}}(x)$,

(ii) or $-\lambda_{0} \notin \Lambda_{1}$ and then $D q_{\lambda_{0}}(x)=0$.

This proves our claim.

In Remark 6.10 we saw that (6.14) along with (5.3) leads to the symmetry of the operator $Q_{\nu}$ expressed by (6.15). However, if $\mu+\lambda \in \Lambda_{1}$ for some $\mu, \lambda \in \Lambda_{1}$, the symmetry of $Q_{\nu}$ may not occur.

Example 6.1. Let $d=1$ and $\Lambda_{1}=\{-3,-1,1,2\}$. Take a smooth function $f(x)$ such that $1 \leq f \leq 2$ and set

$$
q_{-3}=1, \quad q_{-1}=q_{2}=3-f, \quad q_{1}=f .
$$

Then

$$
\sum_{\lambda \in \Lambda_{1}} q_{\lambda} \lambda=-3-(3-f)+f+2(3-f)=0 .
$$

Furthermore,

$$
h^{-1} \sum_{\lambda \in \Lambda_{1}} q_{\lambda}^{\prime} \delta_{\lambda} \varphi=h^{-2} f^{\prime}\left(-T_{-1}+1+T_{1}-T_{2}\right) \varphi=f^{\prime} R \varphi,
$$

where

$$
R:=h^{-2}\left(T_{2}-1\right)\left(T_{-1}-1\right)=\delta_{2} \delta_{-1} .
$$

It follows that (5.3) is satisfied. Also observe that for $\mu \in \Lambda_{1}$ we have

$$
Q_{\mu} \varphi=h^{-1} \sum_{\lambda \in \Lambda_{1}}\left(\delta_{\mu} q_{\lambda}\right) \delta_{\lambda} \varphi=\left(\delta_{\mu} f\right) R \varphi
$$

and

$$
Q_{\mu} T_{\mu} \varphi=Q_{\mu}\left(T_{\mu}-1\right) \varphi+Q_{\mu} \varphi=\sum_{\lambda \in \Lambda_{1}}\left(\delta_{\mu} q_{\lambda}\right) \delta_{\lambda} \delta_{\mu} \varphi+Q_{\mu} \varphi .
$$

This and the fact that $q_{\lambda} \geq 1$ easily imply that condition (5.2) is also satisfied with appropriate constants and operator $\mathcal{K}$ in case there are terms also with $p_{\lambda}$ in $L$ and either $p_{\lambda} \geq 0$ or $h$ is sufficiently small so that $\chi_{\lambda} \geq 1 / 2$.

Remark 6.11. Note that the argument in the above example shows that (always under Assumptions 2.1 and 2.2) an operator

$$
L_{h}=h^{-1} \sum_{\lambda \in \Lambda_{1}} q_{\lambda} \delta_{\lambda}-c
$$

satisfies Assumption 2.3 if $q_{\lambda} \geq \kappa>0$ for a constant $\kappa>0$ and the equality

$$
h^{-1} \sum_{\lambda \in \Lambda_{1}}\left(D_{i} q_{\lambda}\right) \delta_{\lambda}=\sum_{\lambda, \mu \in \Lambda_{1}} q_{i \lambda \mu} \delta_{\lambda} \delta_{\mu}, \quad i=1,2, \ldots, d
$$

holds with some bounded coefficients $q_{i \lambda \mu}$. Therefore it would be useful to find simple conditions, i.e., which can be easily verified, for the characterization of $\Lambda_{1}$ and $q_{\lambda}$ satisfying (6.18). In this direction we have the following condition and conjecture about a criterion for (6.18) to hold.

We call a function $\varphi$ on $\Lambda_{1} \cup\{0\}$ linear if $\varphi(\nu)=\varphi(\lambda)+\varphi(\mu)$ whenever $\nu, \lambda, \mu \in$ $\Lambda_{1} \cup\{0\}$ and $\nu=\mu+\lambda$. 
Conjecture. Equation (6.18) holds with some $q_{i \lambda \mu}$ if and only if

$$
\sum_{\lambda \in \Lambda_{1}}\left(D q_{\lambda}\right) \varphi(\lambda)=0
$$

holds for any $\varphi$ that is linear on $\Lambda_{1} \cup\{0\}$.

Notice that (6.18) and the condition of the conjecture are satisfied, for example, when property (S) holds, or if $\Lambda_{1}$ is the union of disjoint triplets $\{\lambda, \mu, \lambda+\mu\}$ such that $-D q_{\lambda+\mu}=D q_{\lambda}=D q_{\mu}$ for each of them.

Remark 6.12. Condition (5.1) is "fool proof" in two ways related to changes of variables. For simplicity we only concentrate on the case that $\tau_{0}=0$ and $\tau_{\lambda}=1$ for $\lambda \in \Lambda_{1}$. First, one can try changing the time variable by introducing the new function $v(t, x)=u\left(\kappa^{-1} t, x\right)$, where $\kappa>0$ is a constant. This amounts to dividing the coefficients of (2.1) and $f$ by $\kappa$ and accordingly changing the time $t \rightarrow \kappa^{-1} t$. However, as is easy to see, this will not affect condition (5.1) and, for that matter, the value of $F_{1}$ in Theorem 2.1 either.

The second way is to try to relax condition (5.1) by changing the space variable. Introduce

$$
\begin{aligned}
\bar{h} & =\kappa h, \quad S: \psi \rightarrow S \psi(t, x)=\psi\left(t, \kappa^{-1} x\right), \\
\bar{q}_{\lambda} & =\kappa^{2} S q_{\lambda}, \quad \bar{p}_{\lambda}=\kappa S p_{\lambda}, \\
\bar{L}_{\bar{h}}^{0} & =\bar{h}^{-1} \sum_{\lambda \in \Lambda_{1}} \bar{q}_{\lambda} \delta_{\bar{h}, \lambda}+\sum_{\lambda \in \Lambda_{1}} \bar{p}_{\lambda} \delta_{\bar{h}, \lambda}, \\
\bar{L}_{\bar{h}, \mu}^{0} & =\bar{h}^{-1} \sum_{\lambda \in \Lambda_{1}}\left(\delta_{\bar{h}, \mu} \bar{q}_{\lambda}\right) \delta_{\bar{h}, \lambda}+\sum_{\lambda \in \Lambda_{1}}\left(\delta_{\bar{h}_{, \mu}} \bar{p}_{\lambda}\right) \delta_{\bar{h}, \lambda} .
\end{aligned}
$$

One easily checks that

$$
\delta_{\bar{h}, \lambda}=\kappa^{-1} S \delta_{h, \lambda} S^{-1}, \quad \lambda \in \Lambda, \quad \bar{L}_{\bar{h}}^{0}=S L_{h} S^{-1} .
$$

Owing to (6.19), if $u$ satisfies (2.1), then $\bar{u}=S u$ satisfies

$$
\frac{\partial \bar{u}}{\partial t}=\bar{L}_{\bar{h}}^{0} \bar{u}-\bar{c} \bar{u}+\bar{f}
$$

where $\bar{c}=S c, \bar{f}=S f$. Furthermore, $\bar{L}_{\bar{h}, \mu}^{0}=\kappa^{-1} S L_{h, \mu} S^{-1}$ and if (5.1) is satisfied, then for $\psi=S^{-1} \varphi$,

$$
\begin{aligned}
& 2 \sum_{\lambda \in \Lambda_{1}}\left(\delta_{\bar{h}, \lambda} \varphi\right) \bar{L}_{\bar{h}, \lambda}^{0} T_{\bar{h}, \lambda} \varphi=2 \kappa^{-2} S \sum_{\lambda \in \Lambda_{1}}\left(\delta_{h, \lambda} \psi\right) L_{h, \lambda}^{0} T_{h, \lambda} \psi \\
& \quad \leq(1-\delta) \kappa^{-2} S \sum_{\nu \in \Lambda, \lambda \in \Lambda_{1}}\left(q_{\lambda}+h p_{\lambda}\right)\left|\delta_{h, \lambda} \delta_{h, \nu} \psi\right|^{2} \\
& \quad+K_{1} \kappa^{-2} S \sum_{\lambda \in \Lambda_{1}}\left(q_{\lambda}+h p_{\lambda}\right)\left|\delta_{h, \lambda} \psi\right|^{2}+2(1-\delta) \kappa^{-2} S\left[c \mathcal{K}_{h}\left(\sum_{\lambda \in \Lambda}\left|\delta_{h, \lambda} \psi\right|^{2}\right)\right] \\
& \quad=(1-\delta) \sum_{\nu \in \Lambda, \lambda \in \Lambda_{1}}\left(\bar{q}_{\lambda}+\bar{h} \bar{p}_{\lambda}\right)\left|\delta_{\bar{h}, \lambda} \delta_{\bar{h}, \nu} \varphi\right|^{2} \\
& \quad+K_{1} \kappa^{-2} \sum_{\lambda \in \Lambda_{1}}\left(\bar{q}_{\lambda}+\bar{h} \bar{p}_{\lambda}\right)\left|\delta_{\bar{h}, \lambda} \varphi\right|^{2}+2(1-\delta) \bar{c} S \mathcal{K}_{h} S^{-1}\left(\sum_{\lambda \in \Lambda}\left|\delta_{\bar{h}, \lambda} \varphi\right|^{2}\right),
\end{aligned}
$$

where $S \mathcal{K}_{h} S^{-1} \in \mathfrak{K}$. We see that this change of coordinates did not produce any effect on (5.1) apart from changing $K_{1}$ and $\mathcal{K}_{h}$, which is irrelevant. 


\section{REFERENCES}

[1] Hongjie Dong and N.V. Krylov, On the rate of convergence of finite-difference approximations for Bellman equations with constant coefficients, Algebra i Analiz, Vol. 17 (2005), No. 2, 108-132; St. Petersburg Math. J, Vol. 17 (2006), No. 2, 295-313. MR2159586 (2006f:49050)

[2] Hongjie Dong and N.V. Krylov, On the rate of convergence of finite-difference approximations for degenerate linear parabolic equations with $C^{1}$ and $C^{2}$ coefficients, Electron. J. Diff. Eqns., Vol. 2005(2005), No. 102, pp. 1-25. http://ejde.math.txstate.edu MR2162263 (2006i:35008)

[3] Hongjie Dong and N.V. Krylov, On the rate of convergence of finite-difference approximations for parabolic Bellman equations with Lipschitz coefficients in cylindrical domains, Applied Math. and Optimization, Vol. 56 (2007), No. 1, 37-66. MR2334605 (2008e:65240)

[4] I. Gyöngy, Lattice approximations for stochastic quasi-linear parabolic partial differential equations driven by space-time white noise. II, Potential Anal., Vol. 11 (1999), No. 1, 1-37. MR 1699161 (2000g:60106)

[5] I. Gyöngy and N.V. Krylov, Accelerated convergence of finite difference schemes for second order parabolic and elliptic PDEs, in preparation.

[6] N.V. Krylov, A priori estimates of smoothness of solutions to difference Bellman equations with linear and quasi-linear operators, Math. Comp., Vol. 76 (2007), 669-698. MR2291833 (2008e:65250)

[7] N.V. Krylov, On factorizations of smooth nonnegative matrix-values functions and on smooth functions with values in polyhedra, Applied Math. Optimiz., Vol. 58 (2008), No. 3, 373-392. MR2456852

[8] W. Littman, Résolution du problème de Dirichlet par la méthode des différences finies, C. R. Acad. Sci. Paris, Vol. 247 (1958), 2270-2272. MR0107748 (21:6470)

[9] J. von Neumann and R.D. Richtmyer, A method for the numerical calculation of hydrodynamic shocks, J. Appl. Phys., Vol. 21 (1950), 232-237. MR0037613 (12:289b)

[10] Hyek Yoo, Semi-discretization of stochastic partial differential equations on $R^{1}$ by a finite-difference method, Math. Comp., Vol. 69 (2000), No. 230, 653-666. MR1654030 (2000i:65129)

School of Mathematics, University of Edinburgh, King's Bulldings, Edinburgh, EH9 3JZ, United Kingdom

E-mail address: gyongy@maths.ed.ac.uk

127 Vincent Hall, University of Minnesota, Minneapolis, Minnesota, 55455

E-mail address: krylov@math.umn.edu 